\title{
Modeling and Simulation of MIMO Mobile-to-Mobile Wireless Fading Channels
}

\author{
Gholamreza Bakhshi, ${ }^{1}$ Reza Saadat, ${ }^{1}$ and Kamal Shahtalebi ${ }^{2}$ \\ ${ }^{1}$ Communication Research Laboratory, Faculty of Electrical and Computer Engineering, Yazd University, Yazd 89168-69511, Iran \\ ${ }^{2}$ Department of Electrical Engineering, University of Isfahan, Isfahan 81746-73441, Iran \\ Correspondence should be addressed to Kamal Shahtalebi, shahtalebi@eng.ui.ac.ir
}

Received 24 January 2012; Revised 21 March 2012; Accepted 22 March 2012

Academic Editor: Hon Tat Hui

Copyright (C) 2012 Gholamreza Bakhshi et al. This is an open access article distributed under the Creative Commons Attribution License, which permits unrestricted use, distribution, and reproduction in any medium, provided the original work is properly cited.

\begin{abstract}
Analysis and design of multielement antenna systems in mobile fading channels require a model for the space-time cross-correlation among the links of the underlying multipleinput multiple-output (MIMO) Mobile-to-Mobile (M-to-M) communication channels. In this paper, we propose the modified geometrical two-ring model, a MIMO channel reference model for M-to-M communication systems. This model is based on the extension of single-bounce two-ring scattering model for flat fading channel under the assumption that the transmitter and the receiver are moving. Assuming single-bounce scattering model in both isotropic and nonisotropic environment, a closed-form expression for the space-time cross-correlation function (CCF) between any two subchannels is derived. The proposed model provides an important framework in M-to-M system design, where includes many existing correlation models as special cases. Also, two realizable statistical simulation models are proposed for simulating both isotropic and nonisotropic reference model. The realizable simulation models are based on Sum-of-Sinusoids $(\mathrm{SoS})$ simulation model. Finally, the correctness of the proposed simulation models is shown via different simulation scenarios.
\end{abstract}

\section{Introduction}

Mobile-to-Mobile communication channels are expected to play an important role in mobile ad-hoc networks (MANETs), intelligent transportation systems, and relaybased cellular networks, where both the transmitter (Tx) and the receiver $(\mathrm{Rx})$ are in motion. $\mathrm{M}$-to-M channels differ from conventional Base-to-Mobile (B-to-M) cellular radio channels, where the base station (BS) is stationary and relatively free of local scattering.

In a typical macrocell, the BS is elevated and it receives the signal within a narrow beam width, whereas the mobile station (MS) is surrounded by local scatterers. MIMO channel modeling of this typical macrocell environment was investigated in $[1,2]$. However, in outdoor microcells, indoor picocells, and M-to-M communication channels, both Tx $\left(\mathrm{BS} / \mathrm{MS}_{T}\right)$ and $\mathrm{Rx}\left(\mathrm{MS} / \mathrm{MS}_{R}\right)$ are normally surrounded by local scatterers. Clearly, the MIMO macrocell models of $[1,2]$ cannot be used for such environments. For these situations, we need a double-directional channel model (see, e.g., [3$5]$, in which the double-directional concept is introduced and some measurements results are provided). Akki and Haber $[6,7]$ showed that the received envelope on M-to-M channels is Rayleigh faded under non line-of-sight (NLoS) condition, but the statistical properties differ from B-to-M channels. They proposed a reference model for single-input single-output (SISO) M-to-M Rayleigh fading channels. Methods for simulating SISO M-to-M channels have been proposed in $[8,9]$. Recently, Pätzold et al. have proposed a theoretical reference model for narrow-band MIMO M-toM communication channels in [10-12]. This model is based on geometrical "double-bounce two-ring model" (DBTR) and belongs to the class of double-directional channel models. DBTR model assumes that both $\mathrm{Tx}$ and $\mathrm{Rx}$ are surrounded by scatterers and each ray is reflected twice. In the other words, in DBTR model, it is assumed that every Tx side scatterer captures the radio signal from $\mathrm{Tx}$ and reradiates it in the form of a plane wave to the Rx side scatterers. Then, $\mathrm{Rx}$ receives the transmitted radio signal from itself scatterers. A distance-independent DBTR model was proposed in [13] and was simulated in [14]. The main difficulty of DBTR model, discussed in $[15,16]$, is that the signals reflected by 
the scatterers at the Rx side are possibly not independent and the channel coefficient may still not be zero mean complex Gaussian. Therefore, the channel covariance matrix cannot completely describe the MIMO channel [16].

This paper proposes a theoretical reference model for MIMO M-to-M Rayleigh fading channels, avoiding difficulty of DBTR model. This model is based on the extension of geometrical "single-bounce two-ring" (SBTR) model proposed in [17] for MIMO B-to-M channel. The SBTR model belongs to the class of double-directional B-to-M channel models. In [17], the authors have avoided many difficulties of the DBTR model. Furthermore the correctness of their model has been shown via real experimental data. Our model, named here modified geometrical two-ring (MGTR), in comparison with SBTR, includes the mobility of both the transmitter and the receiver. Furthermore, we derive a closed-form spacetime correlation function for 2D nonisotropic scattering environment. Also, we propose two realizable SoS-based simulation models for simulating the reference model in both isotropic and nonisotropic conditions.

The remainder of the paper is organized as follows. In Section 2, we describe the MGTR, a theoretical reference model for MIMO M-to-M channels. In Section 3, a closed-form expression for the space-time cross-correlation function (STCCF) is derived from our theoretical reference model. Section 4 details the SoS-based simulation models. In Section 5, we describe the calculating methods of simulation model's parameters. The comparison of the statistical simulation model with the theoretical reference model is presented in Section 6. Finally, concluding remarks are provided in Section 7.

\section{Theoretical Reference Model for MIMO M-to-M Channels}

In this section, we describe the MGTR model for narrowband MIMO M-to-M channels. As mentioned before, MGTR is based on the extension of SBTR model in [17], in which both transmitter and receiver are in motion. Consider a narrow-band single-user MIMO communication system with $n_{T}$ transmitter and $n_{R}$ receiver antenna elements. Assume both $\mathrm{Tx}$ and $\mathrm{Rx}$ are in motion and equipped with low elevation antennas. The radio propagation environment is characterized by $2 \mathrm{D}$ scattering with NLoS conditions between the transmitter and the receiver. The MIMO channel can be described by an $n_{R} \times n_{T}$ matrix $H(t)=\left[h_{i j}(t)\right]_{n_{R} \times n_{T}}$ of complex faded envelopes.

2.1. Geometrical Modified Two-Ring Model. The geometry of modified two-ring model is shown in Figure 1 for a MIMO M-to-M channel with $n_{T}=n_{R}=2$ antenna elements, where local scatterers of $M S_{T}$ and $M S_{R}$ are distributed on two separate rings. The key difference between our model and M-to-M DBTR model is that here only single-bounce rays are considered while in M-to-M DBTR model double-bounce rays have been considered. Therefore, our assumption avoids the problems of DBTR model. Here, we can model the multiple-bounce rays as secondary effects. As can be seen from Figure 1, the local scatterers around the transmitter, denoted by $S_{T}^{k}\left(k=1,2, \ldots, N_{T}\right)$, are located on a ring of radius $R^{\prime}$, while the local scatterers $S_{R}^{i}\left(i=1,2, \ldots, N_{R}\right)$ around the receiver lie on a separate ring of radius $R$. The symbols $\varphi_{T}$ and $\phi_{R}$ denote the main angle of departure (AOD) and the main angle of arrival (AOA), respectively and the symbols $\varphi_{R}$ and $\phi_{T}$ denote the auxiliary AOD and the auxiliary AOA, respectively. It is assumed that the radii $R^{\prime}$ and $R$ are small in comparison with $D$, which is the distance between the transmitter and the receiver (i.e., $\max \left\{R, R^{\prime}\right\} \ll$ $D)$. The antenna spacings at the transmitter and the receiver are denoted by $\delta_{T}$ and $\delta_{R}$, respectively. Since the antenna spacing are generally small in comparison with the radii $R^{\prime}$ and $R$, we might assume that the inequality " $\min \left\{R, R^{\prime}\right\} \gg$ $\max \left\{\delta_{T}, \delta_{R}\right\} "$ is held. The tilt angles between the $x$-axis and the orientation of the antenna array at the transmitter and the receiver are denoted by $\beta_{T}$ and $\beta_{R}$, respectively. Moreover, it is assumed that the transmitter and the receiver move with speeds $v_{T}$ and $v_{R}$ and in direction determined by the angle of motions $\alpha_{T}$ and $\alpha_{R}$, respectively. Furthermore, $2 \Delta$ is the maximum angle spread at $M S_{T}$, determined by the scattering around $M S_{R}$. Similarly, $2 \Delta^{\prime}$ is the maximum angle spread at $M S_{R}$, determined by the scattering around $M S_{T}$. From Figure 1, it is clear that $\Delta=\arcsin (R / D)$, and $\Delta^{\prime}=$ $\arcsin \left(R^{\prime} / D\right)$. Note that geometry of our proposed model includes many existing geometrical models.

However, it must be noted that it is impossible to derive our reference model by fixing one station ( $\mathrm{Tx}$ or $\mathrm{Rx}$ ) and inserting the relative velocity of $\mathrm{Tx}$ and $\mathrm{Rx}$ into other station ( $\mathrm{Rx}$ or $\mathrm{Tx}$ ) in the previous B-to-M models. Here, we have two independent clusters of the received paths and, it follows two independent clusters of doppler components due to relative velocity of the Tx and $\mathrm{Rx}$.

2.2. Derivation of the Reference Model. In this subsection, we derive the reference model for the MIMO M-to-M channel. In Figure 1 by considering the forward channel (from $M S_{T}$ to $M S_{R}$ ), the $M S_{R}$ receives single-bounce rays from both the scatterer $S_{R}^{i}$ around the $M S_{R}$ and the scatterer $S_{T}^{k}$ around the $M S_{T}$. For the frequency flat, subchannel between the antenna elements $A_{T}^{p}$ and $A_{R}^{l}, h_{l p}(t)$ denotes the time-varying complex baseband equivalent channel gain. Mathematical representation of the superposition of rays at the $A_{R}^{l}$ results in the following expression for the normalized channel gain:

$$
\begin{aligned}
h_{l p}(t)=\sqrt{\frac{\eta_{T}}{N_{T}}} \sum_{k=1}^{N_{T}} \exp \left\{-j \frac{2 \pi}{\lambda}\left(d_{A_{T}^{p} S_{T}^{k}}+d_{S_{T}^{k} A_{R}^{l}}\right)\right. \\
\left.+j \Psi_{T}^{k}+j 2 \pi f_{1}^{k} t\right\} \\
+\sqrt{\frac{\eta_{R}}{N_{R}} \sum_{i=1}^{N_{R}} \exp \left\{-j \frac{2 \pi}{\lambda}\left(d_{A_{T}^{p} S_{R}^{i}}+d_{S_{R}^{i} A_{R}^{l}}\right)\right.} \\
\left.+j \Psi_{R}^{i}+j 2 \pi f_{2}^{i} t\right\},
\end{aligned}
$$

where the first and the second summations correspond to the $M S_{T}$ and $M S_{R}$ rings, respectively. This expression shows the 


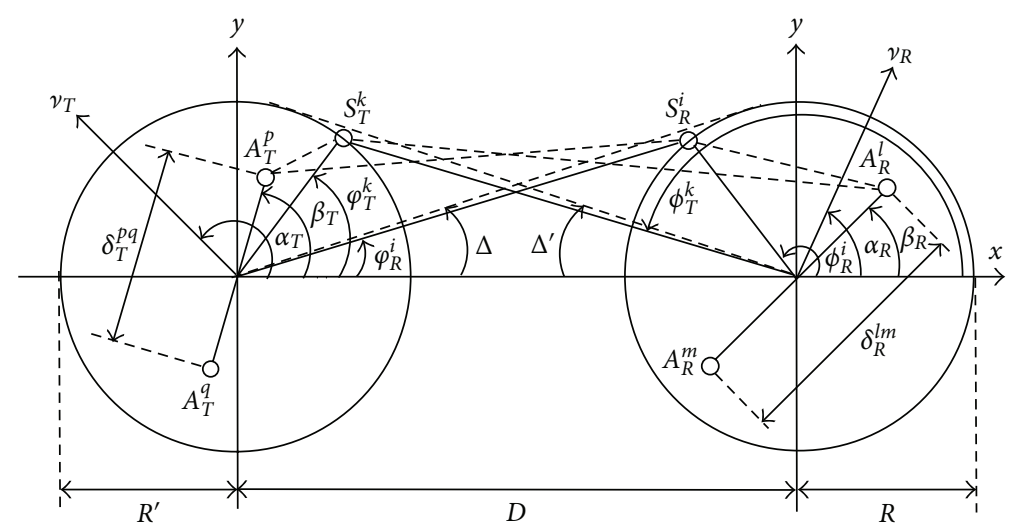

FIGURE 1: The modified geometrical two-ring model for a $2 \times 2$ MIMO channel with scatterers around mobile transmitter $M S_{T}($ left) and mobile receiver $M S_{R}$ (right).

role of AOA and AOD in interrelation between the singlebounce two-ring model in Figure 1 and the $n_{R} \times n_{T}$ channel transfer matrix $H(t)$, in which $h_{l p}(t)$ is the element of row $l$ and column $p$. The $d_{X Y}$ denotes the distance between $X$ and $Y, \eta_{T}$ and $\eta_{R}$ show the respective contributions of scatterers around the $M S_{T}$ and $M S_{R}$ such that $\eta_{T}+\eta_{R}=1 . N_{T}$ and $N_{R}$ are the number of scatterers around the $M S_{T}$ and $M S_{R}$, respectively. $\Psi_{T}^{k}$ and $\Psi_{R}^{i}$ are the associated phase shifts. Furthermore, as shown in Figure 1, $\varphi_{T}^{k}$ and $\varphi_{R}^{i}$ are AoD's of the waves that impinge on $S_{T}^{k}$ and $S_{R}^{i}$. Similarly $\phi_{T}^{k}$ and $\phi_{R}^{i}$ are AoA's of the waves scattered from $S_{T}^{k}$ and $S_{R}^{i}$. Note that $d_{A_{T}^{p} S_{T}^{k}}$ and $d_{A_{T}^{q} S_{T}^{k}}$ are functions of $\varphi_{T}^{k}$, whereas $d_{S_{T}^{k} A_{R}^{l}}$ and $d_{S_{T}^{k} A_{R}^{m}}$ are functions of $\phi_{T}^{k}$. Other $d_{X Y}$ can be easily identified from Figure 1. $\lambda$ is the wavelength and frequencies $f_{1}^{k}$ and $f_{2}^{i}$ are given by

$$
\begin{gathered}
f_{1}^{k}=f_{T_{\max }} \cos \left(\alpha_{T}-\varphi_{T}^{k}\right)+f_{R_{\max }} \cos \left(\alpha_{R}-\phi_{T}^{k}\right), \\
f_{2}^{i}=f_{T_{\max }} \cos \left(\alpha_{T}-\varphi_{R}^{i}\right)+f_{R_{\max }} \cos \left(\alpha_{R}-\phi_{R}^{i}\right),
\end{gathered}
$$

where $f_{T_{\max }}=v_{T} / \lambda$ and $f_{R_{\max }}=v_{R} / \lambda$ are the maximum Doppler frequencies caused by the movement of the transmitter and the receiver, respectively. We also assume $\left\{\Psi_{T}^{k}\right\}_{k=1}^{N_{T}}$ and $\left\{\Psi_{R}^{i}\right\}_{i=1}^{N_{R}}$ are mutually independent and identically distributed (i.i.d) random variables with uniform distributions over $[0,2 \pi)$. According to Figure 1 , while $\varphi_{R}^{i}$ and $\phi_{T}^{k}$ are dependent to $\phi_{R}^{i}$ and $\varphi_{T}^{k}$, respectively, $\phi_{R}^{i}$ and $\varphi_{T}^{k}$ are independent variables. In what follows, we call $\varphi_{T}^{k}$ the $\mathrm{AOD}$, and $\phi_{R}^{i}$ the AOA.

\section{The Space-Time Cross-Correlation Function of the Reference Model}

The STCCF plays an important role in MIMO communication channels. In this section, we derive a closed-form expression for STCCF. The normalized STCC between two subchannel gains $h_{l p}(t)$ and $h_{m q}(t)$ is defined by $\rho_{l p, m q}(\tau)=$ $\mathbb{E}\left[h_{l p}(t) h_{m q}^{*}(t+\tau)\right]$, where $\mathbb{E}(\cdot)$ is the statistical expectation operator and $(\cdot)^{*}$ denotes complex conjugate operation.
Based on independent properties of $\Psi_{T}^{k}$ and $\Psi_{R}^{i}$, it can be asymptotically written by

$$
\begin{aligned}
\rho_{l p, m q}(\tau)= & \lim _{N_{T} \rightarrow \infty} \frac{\eta_{T}}{N_{T}} \sum_{k=1}^{N_{T}} \mathbb{E} \\
& \times\left[\operatorname { e x p } \left\{-j \frac{2 \pi}{\lambda}\left(d_{A_{T}^{p} S_{T}^{k}}-d_{A_{T}^{q} S_{T}^{k}}+d_{S_{T}^{k} A_{R}^{l}}-d_{S_{T}^{k} A_{R}^{m}}\right)\right.\right. \\
& \left.\left.\quad-j 2 \pi f_{1}^{k} \tau\right\}\right] \\
& +\lim _{N_{R} \rightarrow \infty} \frac{\eta_{R}}{N_{R}} \sum_{i=1}^{N_{R}} \mathbb{E} \\
& \times\left[\operatorname { e x p } \left\{-j \frac{2 \pi}{\lambda}\left(d_{A_{T}^{p} S_{R}^{i}}-d_{A_{T}^{q} S_{R}^{i}}+d_{S_{R}^{i} A_{R}^{l}}-d_{S_{R}^{i} A_{R}^{m}}\right)\right.\right. \\
& \left.\left.\quad-j 2 \pi f_{2}^{i} \tau\right\}\right] .
\end{aligned}
$$

For large $N_{T}$ and $N_{R}$, the discrete AoDs, $\varphi_{T}^{k}$, and the discrete AoAs, $\phi_{R}^{i}$, can be replaced with their continuous random variables $\varphi_{T}$ and $\phi_{R}$ with probability density functions (pdf) $f_{M S_{T}}\left(\varphi_{T}\right)$ and $f_{M S_{R}}\left(\phi_{R}\right)$, respectively. Therefore, (4) can be reduced to the following integral form:

$$
\begin{aligned}
& \rho_{l p, m q}(\tau)=\eta_{T} \int_{-\pi}^{\pi} \exp \{-j \frac{2 \pi}{\lambda}\left(d_{A_{T}^{p} S_{T}}-d_{A_{T}^{q} S_{T}}+d_{S_{T} A_{R}^{l}}-d_{S_{T} A_{R}^{m}}\right) \\
&\left.-j 2 \pi f_{1} \tau\right\} f_{M S_{T}}\left(\varphi_{T}\right) d \varphi_{T} \\
&+\eta_{R} \int_{-\pi}^{\pi} \exp \left\{-j \frac{2 \pi}{\lambda}\left(d_{A_{T}^{p} S_{R}}-d_{A_{T}^{q} S_{R}}+d_{S_{R} A_{R}^{l}}-d_{S_{R} A_{R}^{m}}\right)\right. \\
&\left.-j 2 \pi f_{2} \tau\right\} f_{M S_{R}}\left(\phi_{R}\right) d \phi_{R},
\end{aligned}
$$

where $f_{1}$ and $f_{2}$ are the continuous form of $f_{1}^{k}$ and $f_{2}^{i}$ in (2) and (3), respectively. All of the $d_{X Y}$ 's in first integral of (5) depend on $\varphi_{T}$ and in the second integral depend on $\phi_{R}$. 
Based on the application of the law of cosines in appropriate triangles in Figure 1, and assumption $\min \left\{R, R^{\prime}\right\} \gg$ $\max \left\{\delta_{R}, \delta_{T}\right\}$, we obtain the following approximation:

$$
\begin{aligned}
& d_{A_{T}^{p} S_{T}}-d_{A_{T}^{q} S_{T}} \approx-\delta_{T}^{p q} \cos \left(\beta_{T}-\varphi_{T}\right), \\
& d_{S_{T} A_{R}^{l}}-d_{S_{T} A_{R}^{m}} \approx-\delta_{R}^{l m} \cos \left(\beta_{R}-\phi_{T}\right), \\
& d_{A_{T}^{p} S_{R}}-d_{A_{T}^{q} S_{R}} \approx-\delta_{T}^{p q} \cos \left(\beta_{T}-\varphi_{R}\right), \\
& d_{S_{R} A_{R}^{l}}-d_{S_{R} A_{R}^{m}} \approx-\delta_{R}^{l m} \cos \left(\beta_{R}-\phi_{R}\right),
\end{aligned}
$$

Now we apply the law of sines and obtain the following identities:

$$
\begin{gathered}
\frac{D}{\sin \left(\phi_{T}-\varphi_{T}\right)}=\frac{R^{\prime}}{\sin \left(\pi-\phi_{T}\right)}, \\
\frac{D}{\sin \left(\phi_{R}-\varphi_{R}\right)}=\frac{R}{\sin \left(\varphi_{R}\right)} .
\end{gathered}
$$

Based on the assumption $\max \left\{R, R^{\prime}\right\} \ll D$, we conclude that $\Delta \approx R / D$, and $\Delta^{\prime} \approx R^{\prime} / D$. This observation, together with $\sin \epsilon \approx \epsilon$ when $\epsilon$ is small, considering $\phi_{T}$ is almost $\pi$ and $\varphi_{R}$ is almost 0 , allows us to derive the following approximations from (7):

$$
\begin{gathered}
\phi_{T} \approx \pi-\Delta^{\prime} \sin \varphi_{T}, \\
\varphi_{R} \approx \Delta \sin \phi_{R} .
\end{gathered}
$$

Furthermore, using $\sin \epsilon \approx \epsilon$ and $\cos \epsilon \approx 1$ when $\epsilon$ is small, together with (8), the following approximations are derived:

$$
\begin{gathered}
\cos \left(\beta_{R}-\phi_{T}\right) \approx-\cos \beta_{R}+\Delta^{\prime} \sin \beta_{R} \sin \varphi_{T}, \\
\cos \left(\alpha_{R}-\phi_{T}\right) \approx-\cos \alpha_{R}+\Delta^{\prime} \sin \alpha_{R} \sin \varphi_{T}, \\
\cos \left(\beta_{T}-\varphi_{R}\right) \approx \cos \beta_{T}+\Delta \sin \beta_{T} \sin \phi_{R}, \\
\cos \left(\alpha_{T}-\varphi_{R}\right) \approx \cos \alpha_{T}+\Delta \sin \alpha_{T} \sin \phi_{R} .
\end{gathered}
$$

Now, by substituting (10) and (12) to continuous form of (2) and (3), respectively, the following approximations are derived:

$$
\begin{aligned}
& f_{1} \approx f_{T_{\max }} \cos \left(\alpha_{T}-\varphi_{T}\right)-f_{R_{\max }} \cos \alpha_{R}+f_{R_{\max }} \Delta^{\prime} \sin \varphi_{T} \sin \alpha_{R}, \\
& f_{2} \approx f_{T_{\max }} \cos \alpha_{T}+f_{T_{\max }} \Delta \sin \phi_{R} \sin \alpha_{T}+f_{R_{\max }} \cos \left(\alpha_{R}-\phi_{R}\right) .
\end{aligned}
$$

For any given $f_{M S_{T}}(\cdot)$ and $f_{M S_{R}}(\cdot)$, the right-hand side (RHS) of (5) can be calculated numerically, using the trigonometric function relationships given in (6). Note that the RHS of (5) includes two parts. The first part corresponds to STCC contributed by the scattering ring around the $M S_{T}$, and the second part comes from the scattering ring around the $M S_{R}$. Given the assumptions $\max \left\{R, R^{\prime}\right\} \ll D$ and $\min \left\{R, R^{\prime}\right\} \gg \max \left\{\delta_{R}, \delta_{T}\right\}$, by plugging (6), (9) and (11) into (5), equation (5) is approximated by

$$
\begin{gathered}
\rho_{l p, m q}(\tau) \approx \eta_{T} \int_{-\pi}^{\pi} \exp \left\{j \frac { 2 \pi } { \lambda } \left(\delta_{T}^{p q} \cos \left(\beta_{T}-\varphi_{T}\right)+\delta_{R}^{l m}\right.\right. \\
\left.\times\left(-\cos \beta_{R}+\Delta^{\prime} \sin \beta_{R} \sin \varphi_{T}\right)\right) \\
\left.-j 2 \pi f_{1} \tau\right\} f_{M S_{T}}\left(\varphi_{T}\right) d \varphi_{T} \\
+\eta_{R} \int_{-\pi}^{\pi} \exp \left\{j \frac { 2 \pi } { \lambda } \left(\delta_{T}^{p q}\left(\cos \beta_{T}+\Delta \sin \beta_{T} \sin \phi_{R}\right)\right.\right. \\
\left.+\delta_{R}^{l m} \cos \left(\beta_{R}-\phi_{R}\right)\right) \\
\left.-j 2 \pi f_{2} \tau\right\} f_{M S_{R}}\left(\phi_{R}\right) d \phi_{R} .
\end{gathered}
$$

Now, we consider the nonisotropic scattering. Prior works use several different scatterer distributions, included uniform, Gaussian, Laplacian, and von Mises. In this section, we use the von Mises distribution because the measurement experiences show that it approximates many of the previously mentioned distributions. The von Mises pdf is defined by [18]:

$$
p(\theta)=\frac{1}{2 \pi I_{0}(k)} \exp [k \cos (\theta-\mu)]
$$

where $I_{0}(\cdot)$ is the zeroth-order modified Bessel function of the first kind, $\mu \in[-\pi, \pi)$ is the mean angle of scatterers' distribution on the ring, and $k$ controls the spread of scatterers around the mean. When $k=0, p(\theta)=1 /(2 \pi)$ is a uniform distribution yielding $2 \mathrm{D}$ isotropic scattering. As $k$ increases, the scatterers become more clustered around angle $\mu$ and the scattering becomes increasingly nonisotropic. Therefor, the von Mises pdf of AOD and AOA is given by $f_{M S_{T}}\left(\varphi_{T}\right)=\exp \left[k_{T} \cos \left(\varphi_{T}-\mu_{T}\right)\right] /\left(2 \pi I_{0}\left(k_{T}\right)\right)$ and $f_{M S_{R}}\left(\phi_{R}\right)=$ $\exp \left[k_{R} \cos \left(\phi_{R}-\mu_{R}\right)\right] /\left(2 \pi I_{0}\left(k_{R}\right)\right)$, respectively.

From [[19], eq. 3.338], we have

$$
\int_{-\pi}^{\pi} \exp (x \sin \theta+y \cos \theta) d \theta=2 \pi I_{0}\left(\sqrt{x^{2}+y^{2}}\right) .
$$

Under nonisotropic conditions, and by substituting (13) into (14) and calculating the two integrals of (14) by (16), the STCCF of our reference model is derived after some algebraic manipulations (see (17)).

$$
\begin{aligned}
\rho_{l p, m q}(\tau) \approx & \frac{\eta_{T}}{I_{0}\left(k_{T}\right)} \exp \left[-j \frac{2 \pi}{\lambda} \delta_{R}^{l m} \cos \beta_{R}+j 2 \pi f_{R_{\max }} \tau \cos \alpha_{R}\right] \\
& \times I_{0}\left(\left\{k_{T}^{2}-\left(\frac{2 \pi}{\lambda}\right)^{2} \delta_{R}^{l m} \Delta^{\prime} \sin \beta_{R}\left(\delta_{R}^{l m} \Delta^{\prime} \sin \beta_{R}+2 \delta_{T}^{p q} \sin \beta_{T}\right)-\frac{(2 \pi)^{2}}{\lambda} \delta_{T}^{p q}\left(\frac{\delta_{T}^{p q}}{\lambda}-2 f_{T_{\max }} \tau \cos \left(\alpha_{T}-\beta_{T}\right)\right)\right.\right.
\end{aligned}
$$




$$
\begin{aligned}
&-\left(2 \pi f_{T_{\max }} \tau\right)^{2}+2 \frac{(2 \pi)^{2}}{\lambda} \delta_{R}^{l m} \Delta^{\prime} \tau \sin \beta_{R}\left(f_{T_{\max }} \sin \alpha_{T}+\Delta^{\prime} f_{R_{\max }} \sin \alpha_{R}\right)-(2 \pi)^{2} \\
& \times f_{R_{\max }} \tau \Delta^{\prime} \sin \alpha_{R}\left(f_{R_{\max }} \tau \Delta^{\prime} \sin \alpha_{R}-\frac{2}{\lambda} \delta_{T}^{p q} \sin \beta_{T}+2 f_{T_{\max }} \tau \sin \alpha_{T}\right) \\
&\left.\left.+j 2 k_{T}\left[\frac{2 \pi}{\lambda} \delta_{T}^{p q} \cos \left(\beta_{T}-\mu_{T}\right)-2 \pi f_{T_{\max }} \tau \cos \left(\alpha_{T}-\mu_{T}\right)+\frac{2 \pi}{\lambda} \delta_{R}^{l m} \Delta^{\prime} \sin \beta_{R} \sin \mu_{T}-2 \pi f_{R_{\max }} \tau \Delta^{\prime} \sin \alpha_{R} \sin \mu_{T}\right]\right\}^{1 / 2}\right) \\
&+\frac{\eta_{R}}{I_{0}\left(k_{R}\right)} \exp \left[j \frac{2 \pi}{\lambda} \delta_{T}^{p q} \cos \beta_{T}-j 2 \pi f_{T_{\max }} \tau \cos \alpha_{T}\right] \\
& \times I_{0}\left(\left\{k_{R}^{2}-\left(\frac{2 \pi}{\lambda}\right)^{2} \delta_{T}^{p q} \Delta \sin \beta_{T}\left(\delta_{T}^{p q} \Delta \sin \beta_{T}+2 \delta_{R}^{l m} \sin \beta_{R}\right)\right.\right. \\
& \quad-\frac{(2 \pi)^{2}}{\lambda} \delta_{R}^{l m}\left(\frac{\delta_{R}^{l m}}{\lambda}-2 f_{R_{\max }} \tau \cos \left(\alpha_{R}-\beta_{R}\right)\right)-\left(2 \pi f_{R_{\max }} \tau\right)^{2}+2 \frac{(2 \pi)^{2}}{\lambda} \delta_{T}^{p q} \Delta \tau \times \sin \beta_{T}\left(f_{R_{\max }} \sin \alpha_{R}+\Delta f_{T_{\max }} \sin \alpha_{T}\right) \\
& \quad-(2 \pi)^{2} f_{T_{\max }} \tau \Delta \sin \alpha_{T}\left(f_{T_{\max }} \tau \Delta \sin \alpha_{T}-\frac{2}{\lambda} \delta_{R}^{l m} \sin \beta_{R}+2 f_{R_{\max }} \tau \sin \alpha_{R}\right) \\
&\left.\left.\quad+j 2 k_{R}\left[\frac{2 \pi}{\lambda} \delta_{R}^{l m} \cos \left(\beta_{R}-\mu_{R}\right)-2 \pi f_{R_{\max }} \tau \cos \left(\alpha_{R}-\mu_{R}\right)+\frac{2 \pi}{\lambda} \delta_{T}^{p q} \Delta \sin \beta_{T} \sin \mu_{R}-2 \pi f_{T_{\max }} \tau \Delta \sin \alpha_{T} \sin \mu_{R}\right]\right\}^{1 / 2}\right) .
\end{aligned}
$$

Note that many existing correlation functions are special cases of our MIMO M-to-M space-time correlation function in (17). For example: (i) For 2-D isotropic scattering around both $M S_{T}$ and $M S_{R}\left(k_{T}=k_{R}=0\right)$, the STCCF of our reference model reduces to STCCF of MGTR model in isotropic environment [20] as

$$
\begin{aligned}
& \rho_{l p, m q}(\tau) \approx \eta_{T} \exp \left[-j \frac{2 \pi}{\lambda} \delta_{R}^{l m} \cos \beta_{R}+j 2 \pi f_{R_{\max }} \tau \cos \alpha_{R}\right] \\
& \times I_{0}\left(\left\{-\left(\frac{2 \pi}{\lambda}\right)^{2} \delta_{R}^{l m} \Delta^{\prime} \sin \beta_{R}\left(\delta_{R}^{l m} \Delta^{\prime} \sin \beta_{R}+2 \delta_{T}^{p q} \sin \beta_{T}\right)\right.\right. \\
&-\frac{(2 \pi)^{2}}{\lambda} \delta_{T}^{p q}\left(\frac{\delta_{T}^{p q}}{\lambda}-2 f_{T_{\max }} \tau \cos \left(\alpha_{T}-\beta_{T}\right)\right)-\left(2 \pi f_{T_{\max }} \tau\right)^{2}+2 \frac{(2 \pi)^{2}}{\lambda} \delta_{R}^{l m} \Delta^{\prime} \tau \sin \beta_{R}\left(f_{T_{\max }} \sin \alpha_{T}+\Delta^{\prime} f_{R_{\max }} \sin \alpha_{R}\right) \\
&\left.\left.-(2 \pi)^{2} f_{R_{\max }} \tau \Delta^{\prime} \sin \alpha_{R}\left(f_{R_{\max }} \tau \Delta^{\prime} \sin \alpha_{R}-\frac{2}{\lambda} \delta_{T}^{p q} \sin \beta_{T}+2 f_{T_{\max }} \tau \sin \alpha_{T}\right)\right\}^{1 / 2}\right) \\
&+ \eta_{R} \exp \left[j \frac{2 \pi}{\lambda} \delta_{T}^{p q} \cos \beta_{T}-j 2 \pi f_{T_{\max }} \tau \cos \alpha_{T}\right] \\
& \times I_{0}\left(\left\{-\left(\frac{2 \pi}{\lambda}\right)^{2} \delta_{T}^{p q} \Delta \sin \beta_{T}\left(\delta_{T}^{p q} \Delta \sin \beta_{T}+2 \delta_{R}^{l m} \sin \beta_{R}\right)\right.\right. \\
& \quad-\frac{(2 \pi)^{2}}{\lambda} \delta_{R}^{l m}\left(\frac{\delta_{R}^{l m}}{\lambda}-2 f_{R_{\max }} \tau \cos \left(\alpha_{R}-\beta_{R}\right)\right)-\left(2 \pi f_{R_{\max }} \tau\right)^{2}+2 \frac{(2 \pi)^{2}}{\lambda} \delta_{T}^{p q} \Delta \tau \sin \beta_{T}\left(f_{R_{\max }} \sin \alpha_{R}+\Delta f_{T_{\max }} \sin \alpha_{T}\right) \\
&\left.\left.\quad-(2 \pi)^{2} f_{T_{\max }} \tau \Delta \sin \alpha_{T}\left(f_{T_{\max }} \tau \Delta \sin \alpha_{T}-\frac{2}{\lambda} \delta_{R}^{l m} \sin \beta_{R}+2 f_{R_{\max }} \tau \sin \alpha_{R}\right)\right\}^{1 / 2}\right) .
\end{aligned}
$$


(ii) For stationary $M S_{T}\left(f_{T_{\max }}=0\right)$, the STCCF of our reference model reduces to MIMO B-to-M communication channel model based on single-bounce tworing model proposed in [17, equation (7)].

(iii) If there is no scattering around the $M S_{T}$ such in a macrocell $\left(\eta_{T}=0\right)$ and stationary $M S_{T}\left(f_{T_{\max }}=0\right)$, (17) is simplified to STCCF of the conventional "onering" model for MIMO B-to-M communication channel proposed in [2]. In these conditions, the first half of (17) disappears, and the remaining part is the same as (12) in [2].

(iv) If there is no scattering around the $M S_{T}$ such in a macrocell $\left(\eta_{T}=0\right)$, stationary $M S_{T}\left(f_{T_{\max }}=0\right)$ and, with $l=m$ and $p=q$, our reference model's STCCF is simplified to conventional "one-ring" model for SISO B-to-M communication channel. This reduces (17) to the well-known Clarke's temporal correlation function, that is, $J_{0}\left(2 \pi f_{R_{\max }} \tau\right)[21]$, where $J_{0}(\cdot)$ is the Bessel function of the first kind of zero order.

\section{The Simulation Model}

In this section, we derive a statistical simulation model. The theoretical model proposed in Section 2 assumes an infinite number of scatterers, which prevents practical implementation. Actually, in a practical communication channels, the number of scatterers is finite. In the following, we propose a SoS-based statistical simulation model that matches the statistical properties of the theoretical reference model.

Generally, SoS models [22] approximate the underlying random processes by the superposition of a finite number of properly selected functions and can be classified as either statistical or deterministic. In other words, the SoS models are based on a superposition of an infinite number of weighted harmonic functions with equidistant frequencies and random phases. Actually, the SoS models are applied by using only a finite number of harmonic functions for simulating the communication channels [22]. Deterministic SoS models have sinusoids with fixed phases, amplitudes, and Doppler frequencies for all simulation trials. Statistical SoS models leave at least one of the parameter sets (amplitudes, phases, or Doppler frequencies) as random variables that vary with each simulation trial.

The following function is considered as the complexfaded envelope in a real environment that contains finite number of scatterers (finite number of harmonic functions):

$$
\begin{aligned}
& \hat{h}_{l p}(t)=\sqrt{\frac{\eta_{T}}{N_{T}}} \sum_{k=1}^{N_{T}} \exp \left\{-j \frac{2 \pi}{\lambda}\left(d_{A_{T}^{p} S_{T}^{k}}+d_{S_{T}^{k} A_{R}^{l}}\right)\right. \\
& \left.+j \Psi_{T}^{k}+j 2 \pi f_{1}^{k} t\right\} \\
& +\sqrt{\frac{\eta_{R}}{N_{R}}} \sum_{i=1}^{N_{R}} \exp \left\{-j \frac{2 \pi}{\lambda}\left(d_{A_{T}^{p} S_{R}^{i}}+d_{S_{R}^{i} A_{R}^{l}}\right)\right. \\
& \left.+j \Psi_{R}^{i}+j 2 \pi f_{2}^{i} t\right\},
\end{aligned}
$$

where the parameters of above equation are defined in Section 2.2. In contrast to the reference model, the discrete AODs $\varphi_{T}^{k}$ and AOAs $\phi_{R}^{i}$ are now constant, which will be determined in Section 5. The phases $\Psi_{T}^{k}$ and $\Psi_{R}^{i}$ are still i.i.d. random variables, each with uniform distribution on the interval $[0,2 \pi)$. Hence, $\hat{h}_{l p}(t)$ represents a stochastic process. The STCCF between $\hat{h}_{l p}(t)$ and $\hat{h}_{m q}(t)$ is defined as $\hat{\rho}_{l p, m q}(\tau)=\mathbb{E}\left[\hat{h}_{l p}(t) \hat{h}_{m q}^{*}(t+\tau)\right]$, where $(\cdot)^{*}$ denotes the complex conjugate operation, and $\mathbb{E}(\cdot)$ is the statistical expectation operator, which applies to the random phases $\Psi_{T}^{k}$ and $\Psi_{R}^{i}$. It can be shown that STCCF can be expressed in closed form, considering finite scatterers around the $M S_{T}$ and $M S_{R}$ (finite number of harmonic functions), as

$$
\begin{aligned}
\hat{\rho}_{l p, m q}(\tau) \approx & \frac{\eta_{T}}{N_{T}} \sum_{k=1}^{N_{T}} \exp \\
& \times\left\{j \frac { 2 \pi } { \lambda } \left(\delta_{T}^{p q} \cos \left(\beta_{T}-\varphi_{T}^{k}\right)+\delta_{R}^{l m}\right.\right. \\
& \left.\left.\times\left(-\cos \beta_{R}+\Delta^{\prime} \sin \beta_{R} \sin \varphi_{T}^{k}\right)\right)-j 2 \pi f_{1}^{k} \tau\right\} \\
+ & \frac{\eta_{R}}{N_{R}} \sum_{i=1}^{N_{R}} \exp \left\{j \frac { 2 \pi } { \lambda } \left(\delta_{T}^{p q}\left(\cos \beta_{T}+\Delta \sin \beta_{T} \sin \phi_{R}^{i}\right)\right.\right. \\
& \left.\left.+\delta_{R}^{l m} \cos \left(\beta_{R}-\phi_{R}^{i}\right)\right)-j 2 \pi f_{2}^{i} \tau\right\},
\end{aligned}
$$

where

$$
\begin{gathered}
f_{1}^{k} \approx f_{T_{\max }} \cos \left(\alpha_{T}-\varphi_{T}^{k}\right)-f_{R_{\max }} \cos \alpha_{R} \\
+f_{R_{\max }} \Delta^{\prime} \sin \varphi_{T}^{k} \sin \alpha_{R} \\
f_{2}^{i} \approx f_{T_{\max }} \cos \alpha_{T}+f_{T_{\max }} \Delta \sin \phi_{R}^{i} \sin \alpha_{T} \\
+f_{R_{\max }} \cos \left(\alpha_{R}-\phi_{R}^{i}\right) .
\end{gathered}
$$

In the following section we introduce two methods for determining the constant discrete AODs $\varphi_{T}^{k}$ and AOAs $\phi_{R}^{i}$.

\section{Parameters Calculation of Simulation Model}

In this section, we present two methods for the computation of the parameters determining the statistics of the MIMO channel simulation model. The first method is the method of exact Doppler spread (MEDS), which is recommended in case of isotropic scattering. The second method is the $L_{p}$-Norm method. This method can be applied for any given distribution of the local scatterers, such as the Gaussian distribution, the Laplacian distribution, and the von Mises distribution. In other words, the $L_{p}$-Norm method is a general method for calculation of the parameters of deterministic simulation models.

5.1. Method of Exact Doppler Spread (MEDS). The MEDS method was first time proposed in [23], which is recommended in case of isotropic scattering, and was also 


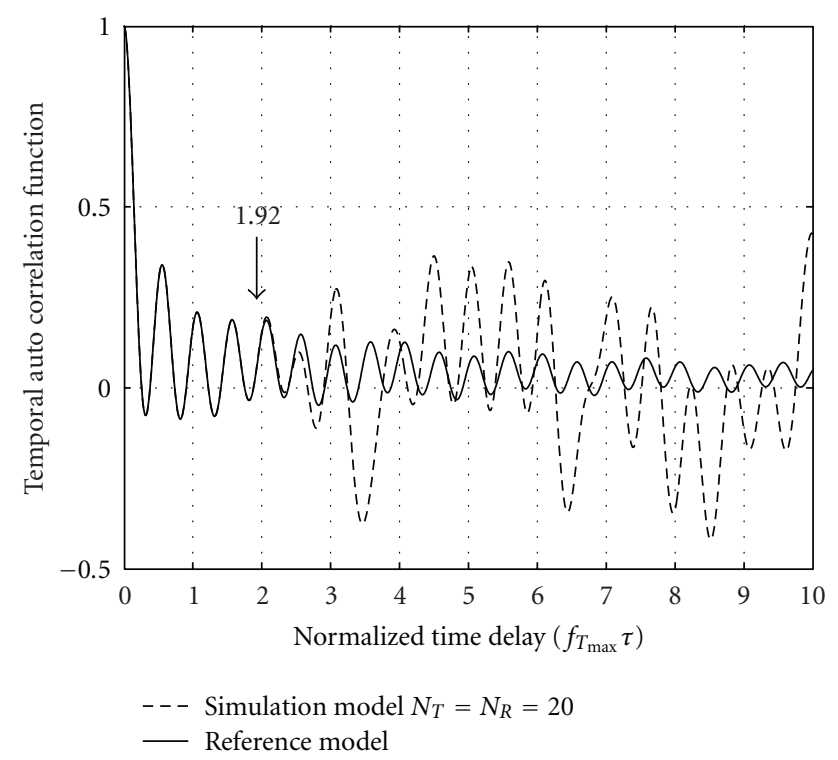

(a)

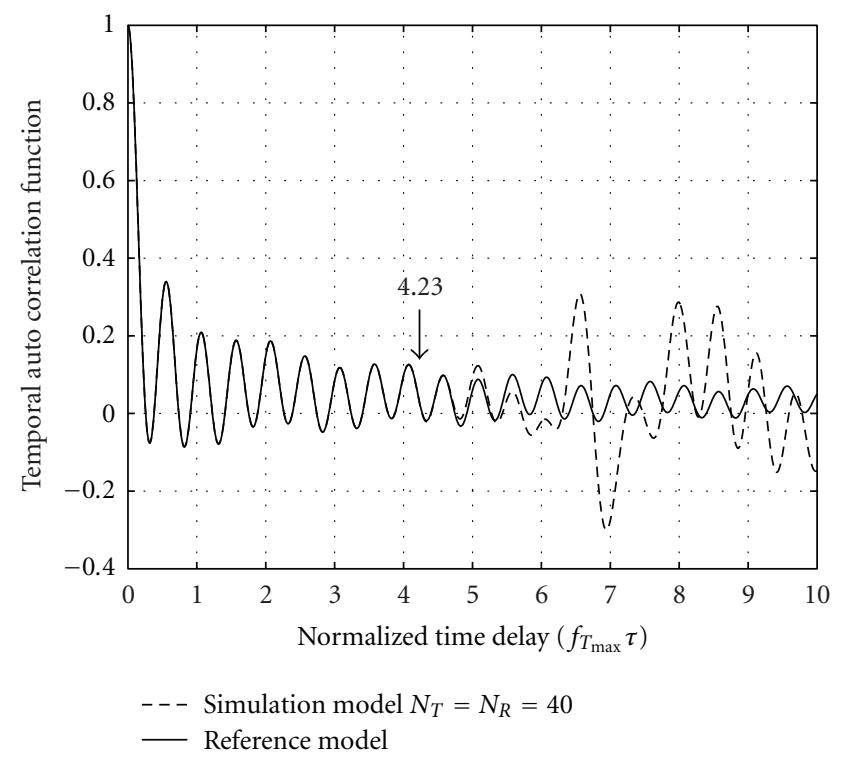

(c)

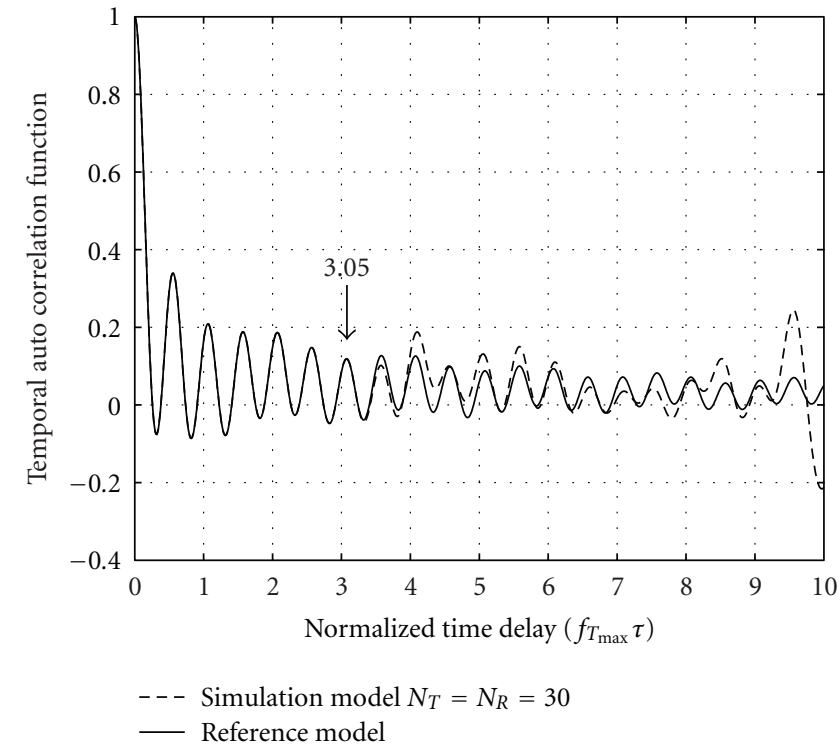

(b)

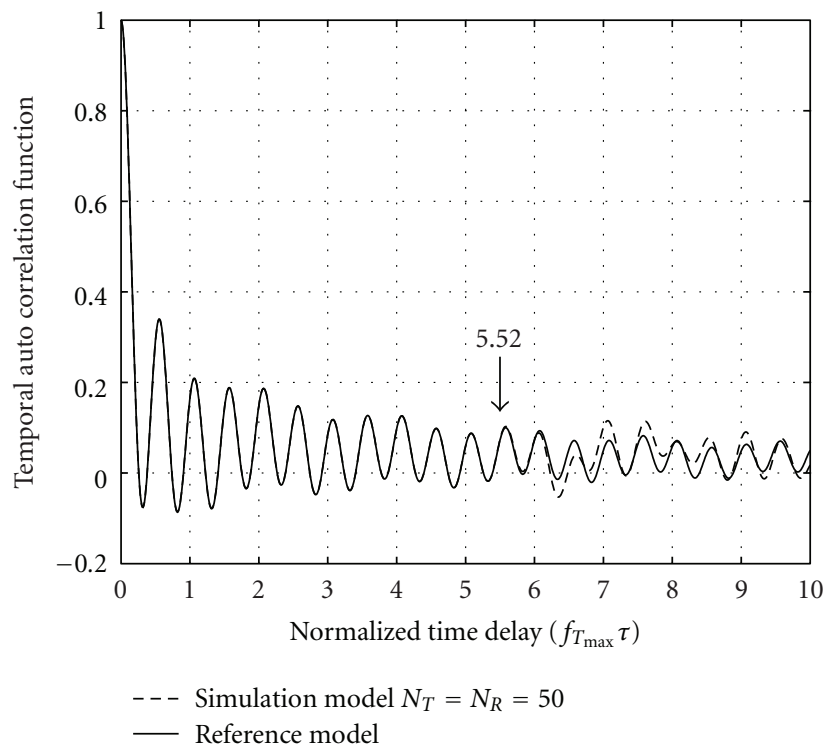

(d)

FIGURE 2: The normalized temporal ACF of the isotropic reference model and the MEDS simulation model for $N_{T}=N_{R}=20,30,40,50$.

described in [22] in details. This method is extended in [11, $12,14]$ for simulating the MIMO M-to-M DBTR reference model. According to MEDS method the discrete AODs $\varphi_{T}^{k}$ and AOAs $\phi_{R}^{i}$ are determined by [22]:

$$
\begin{array}{cl}
\varphi_{T}^{k} & =\frac{2 \pi}{N_{T}}\left(k-\frac{1}{2}\right), \quad k=1,2, \ldots, N_{T}, \\
\phi_{R}^{i} & =\frac{2 \pi}{N_{R}}\left(i-\frac{1}{2}\right), \quad i=1,2, \ldots, N_{R} .
\end{array}
$$

Therefore, in the statistical simulation model only phases $\Psi_{T}^{k}$ and $\Psi_{R}^{i}$ are random parameters. They are i.i.d. random variables uniformly distributed over $[0,2 \pi)$.
5.2. $L_{p}$-Norm Method. When the AODs $\varphi_{T}^{k}$ and AOAs $\phi_{R}^{i}$ are nonuniformly distributed on rings around the transmitter and the receiver, respectively, the recommend method for determining the AODs and AOAs is $L_{p}$-Norm which is described in detail in [22]. This method is extended in [11, $12,14]$ for simulating the MIMO M-to-M DBTR reference model in nonisotropic environment. According to $L_{p}$-Norm method, the discrete AODs $\varphi_{T}^{k}$ and AOAs $\phi_{R}^{i}$ are determined by minimizing the following error norm [22]:

$$
E_{\rho_{l, m q}}^{(p)}=\left\{\frac{1}{\tau_{\max }} \int_{0}^{\tau_{\max }}\left|\rho_{l p, m q}(\tau)-\hat{\rho}_{l p, m q}(\tau)\right|^{p} d \tau\right\}^{1 / p}
$$




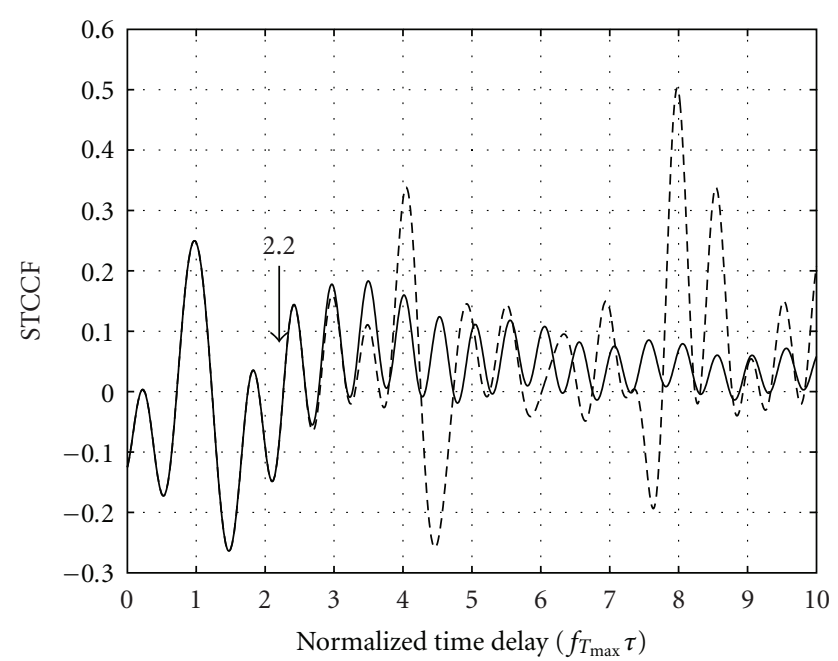

- - Simulation model $N_{T}=N_{R}=20$

— Reference model

(a)

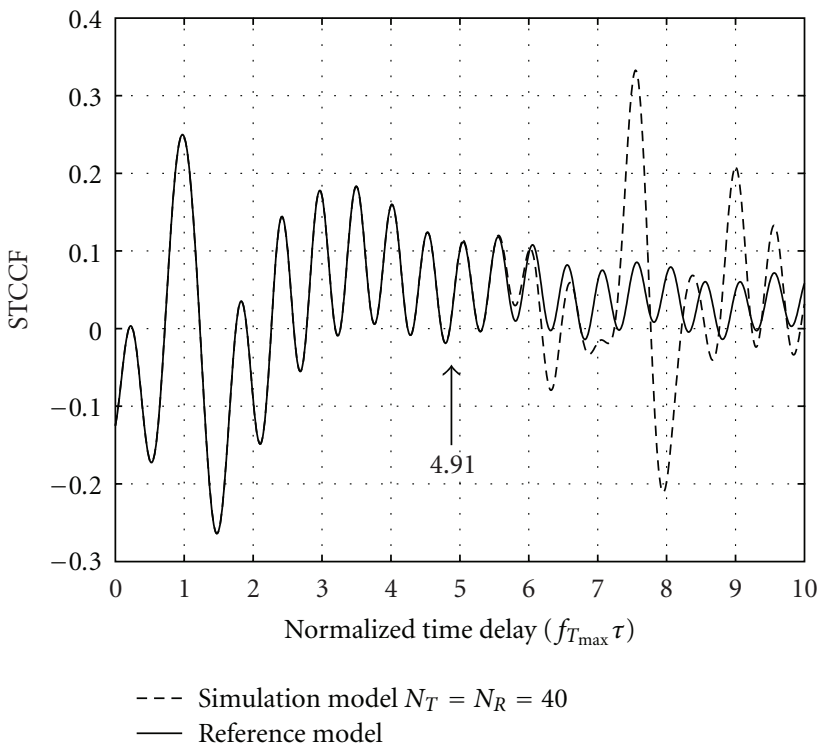

(c)

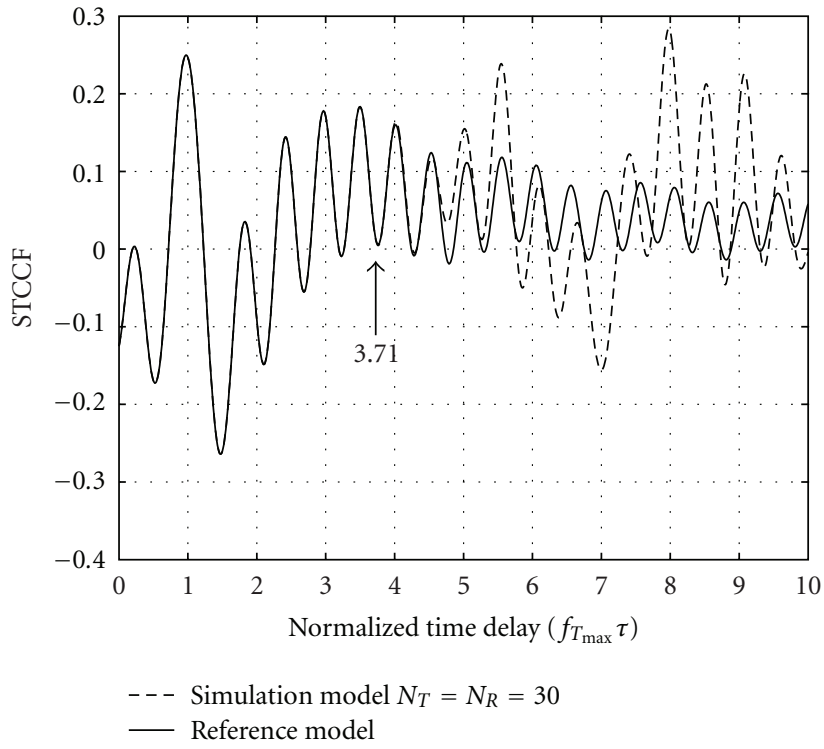

(b)

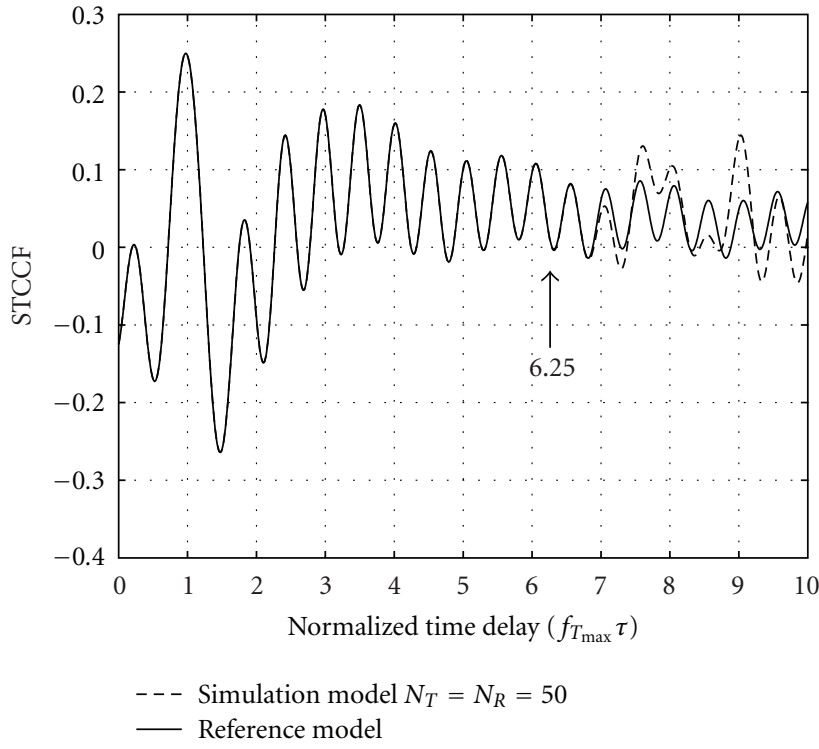

(d)

FIgURE 3: The STCCF of the isotropic reference model and the MEDS simulation model for $\delta_{T}^{p q}=\delta_{R}^{l m}=1 \lambda$ and $N_{T}=N_{R}=20,30,40,50$.

where $p=1,2, \ldots, \rho_{l p, m q}(\tau)$ and $\hat{\rho}_{l p, m q}(\tau)$ are the STCCF of the theoretical reference model in nonisotropic condition (17) and the $L_{p}$-Norm simulation model, respectively. Therefore, in the statistical simulation model only phases $\Psi_{T}^{k}$ and $\Psi_{R}^{i}$ are random parameters. They are i.i.d random variables uniformly distributed over $[0,2 \pi)$.

\section{Performance Evaluation of the Simulation Models}

This section evaluates the performance of the simulation models by comparing its statistical properties with those of the theoretical model. In all simulations, The following parameters were chosen for both models. The antenna tilt angles $\beta_{T}$ and $\beta_{R}$ were defined as $\beta_{T}=\beta_{R}=\pi / 2$. At the transmitter side, the angle of motion $\alpha_{T}$ was set to $\pi / 4$, while the receiver was moving at an angle of $\alpha_{R}=0$. Identical maximum Doppler frequencies $f_{T_{\max }}=f_{R_{\max }}=91 \mathrm{~Hz}$ were assumed, and the wavelength $\lambda$ was set to $\lambda=0.15 \mathrm{~m}$ (according to $[10,11])$. Furthermore, the other parameters have their quantity as $\Delta=\pi / 3, \Delta^{\prime}=\pi / 6, \mu_{T}=5 \pi / 8, \mu_{R}=0$, and $\eta_{R}=0.2$, according to Table I of [17].

6.1. The MEDS Simulation Model. Such as mentioned before, the model parameters $\varphi_{T}^{k}$ and $\phi_{R}^{i}$ have been determined by the MEDS method, since we assume isotropic scattering 


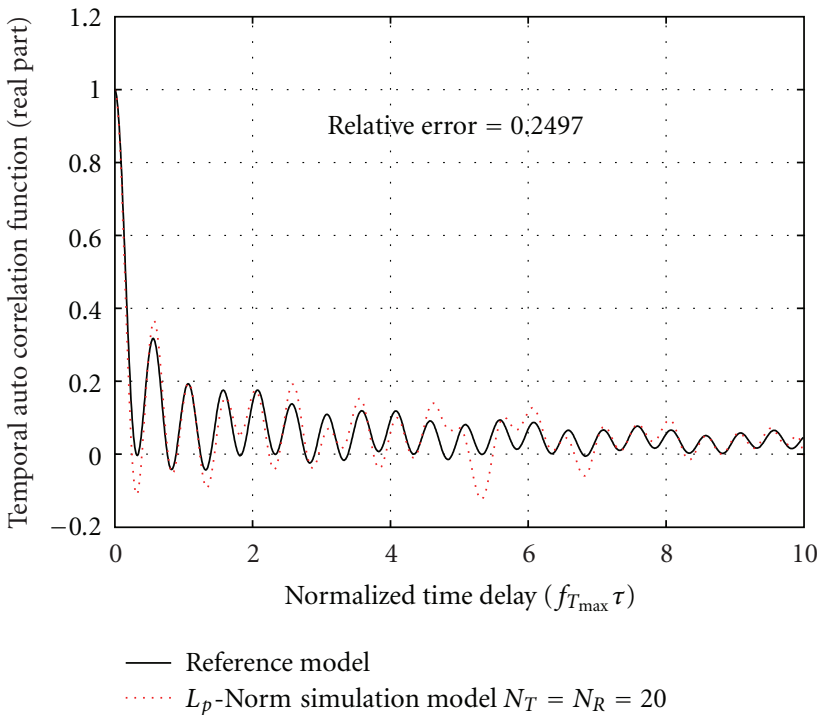

(a)

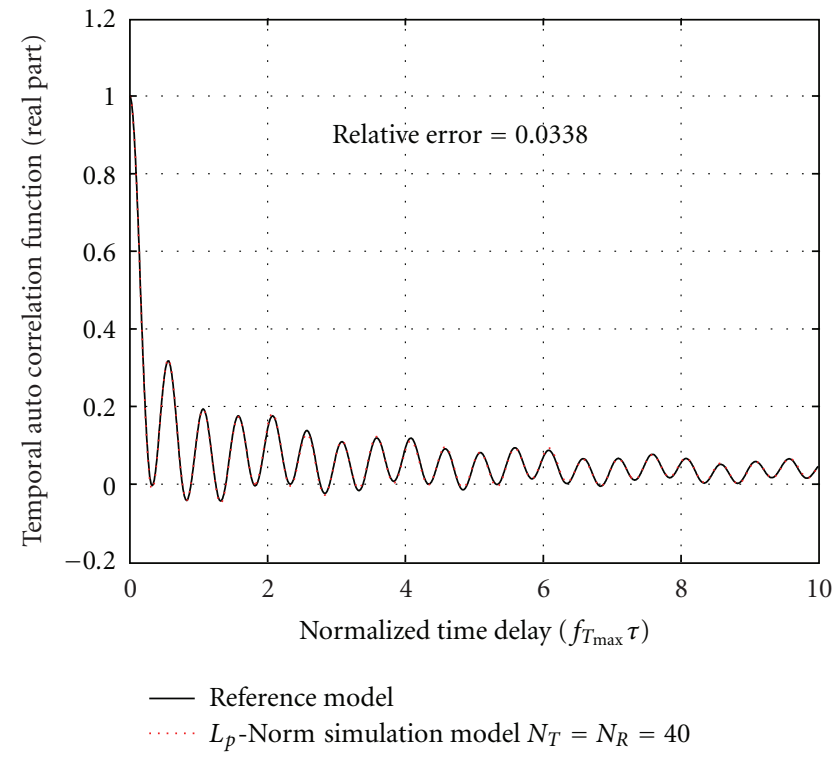

(c)

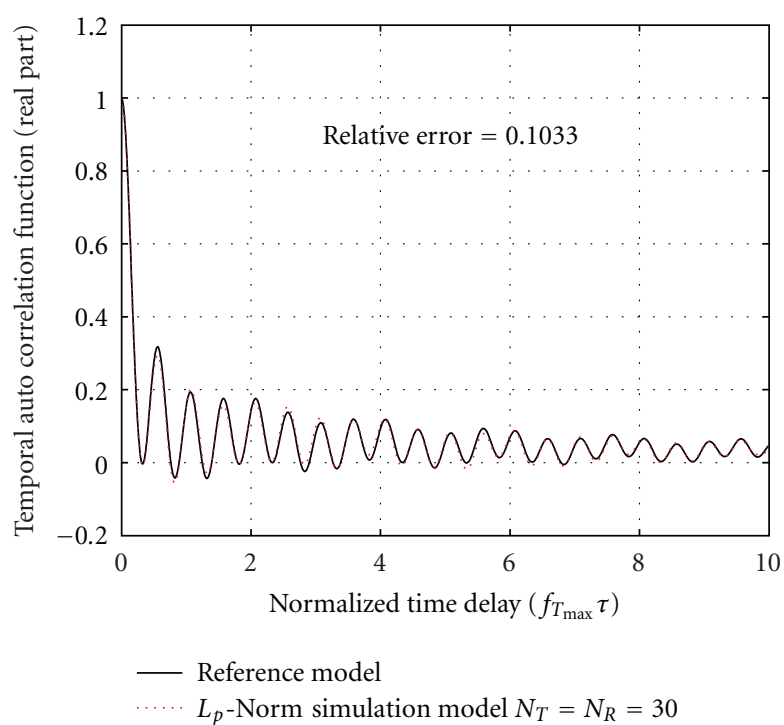

(b)

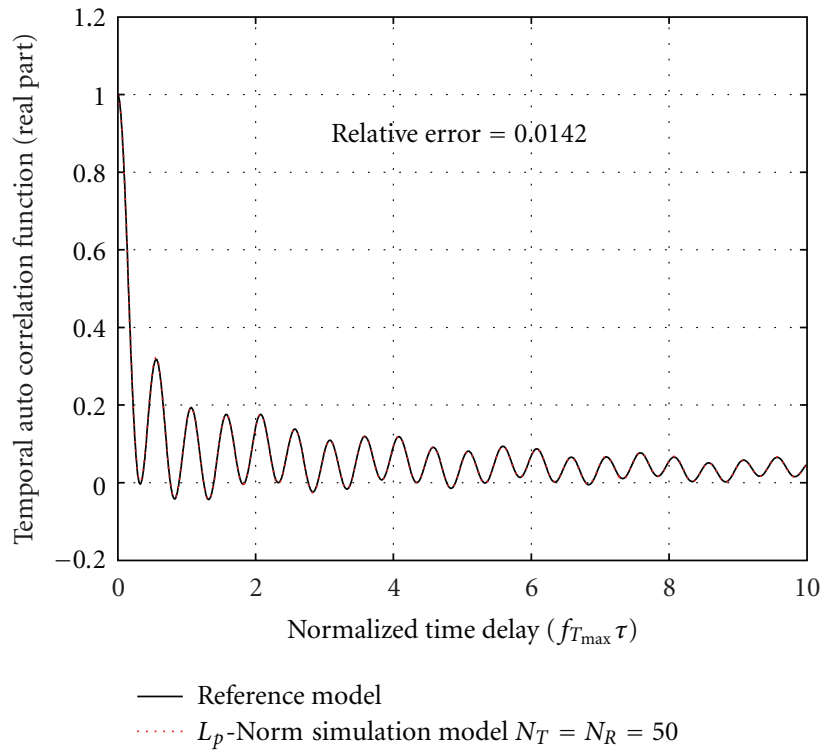

(d)

Figure 4: The normalized temporal ACF of the nonisotropic reference model and the $L_{p}$-Norm simulation model for $N_{T}=N_{R}=20,30,40$, 50 (real part).

around both the transmitter and the receiver $\left(k_{T}=k_{R}=0\right)$. Note that the mismatch criteria in the following simulation is Relative Error and is set to $10^{-3}$. The relative error is defined by

$$
\epsilon_{\rho_{l p, m q}}=\frac{\left\{\left(1 / \tau_{\max }\right) \int_{0}^{\tau_{\max }}\left|\rho_{l p, m q}(\tau)-\hat{\rho}_{l p, m q}(\tau)\right|^{2} d \tau\right\}^{1 / 2}}{\left\{\left(1 / \tau_{\max }\right) \int_{0}^{\tau_{\max }}\left|\rho_{l p, m q}(\tau)\right|^{2} d \tau\right\}^{1 / 2}},
$$

where $\rho_{l p, m q}(\tau)$ and $\hat{\rho}_{l p, m q}(\tau)$ are the STCCF of the theoretical reference model and the simulation model, respectively. Now we consider two simulation scenario as follows. (i) First Scenario. In this scenario, we compare the temporal autocorrelation function (ACF) of the MEDS simulation model with the temporal ACF of theoretical isotropic reference model, derived in Section 3 (18). Figure 2 shows this comparison for $\left\{N_{T}=N_{R}=20,30,40,50\right\}$. This figure shows that the temporal ACF of the MEDS simulation model is matched to the temporal ACF of theoretical isotropic reference model until a limited normalized time delay, that is shown in the subfigures and we call it the Matched Time. In other words, the matched time is the maximum normalized time delay that until it relative error between the reference and the 


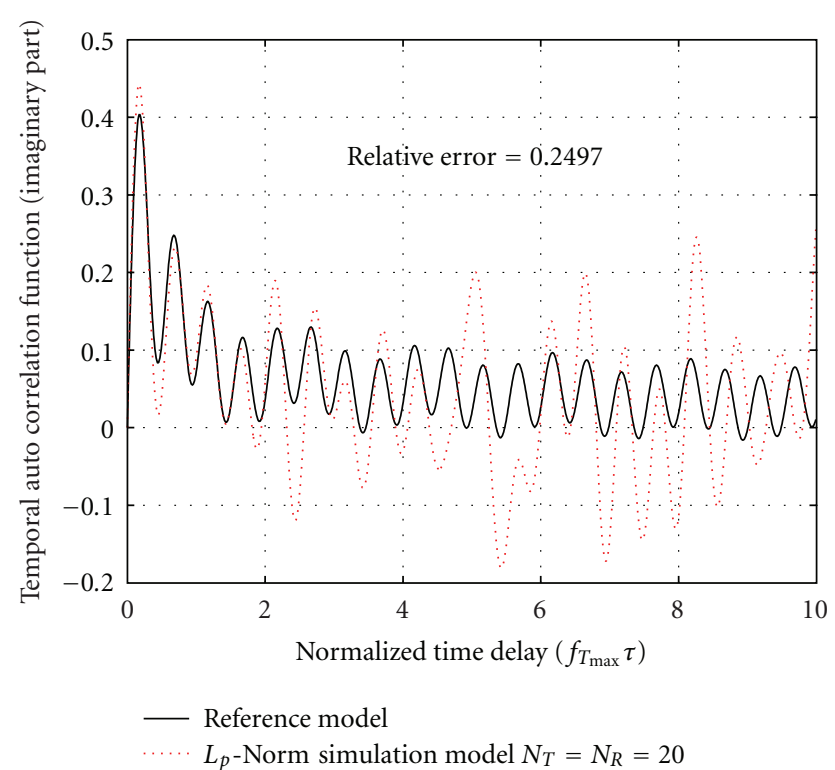

(a)

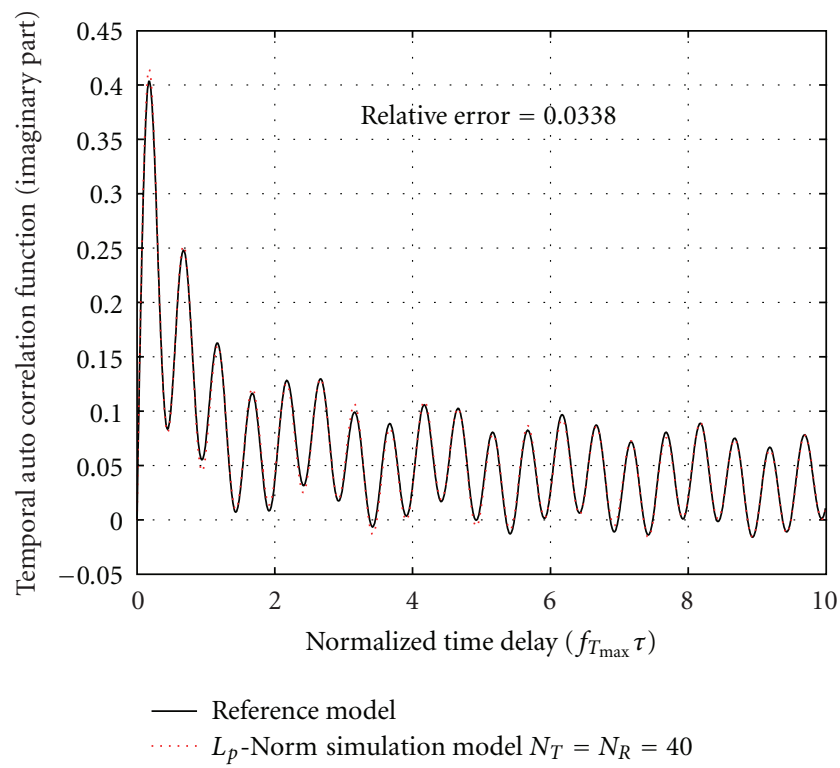

(c)

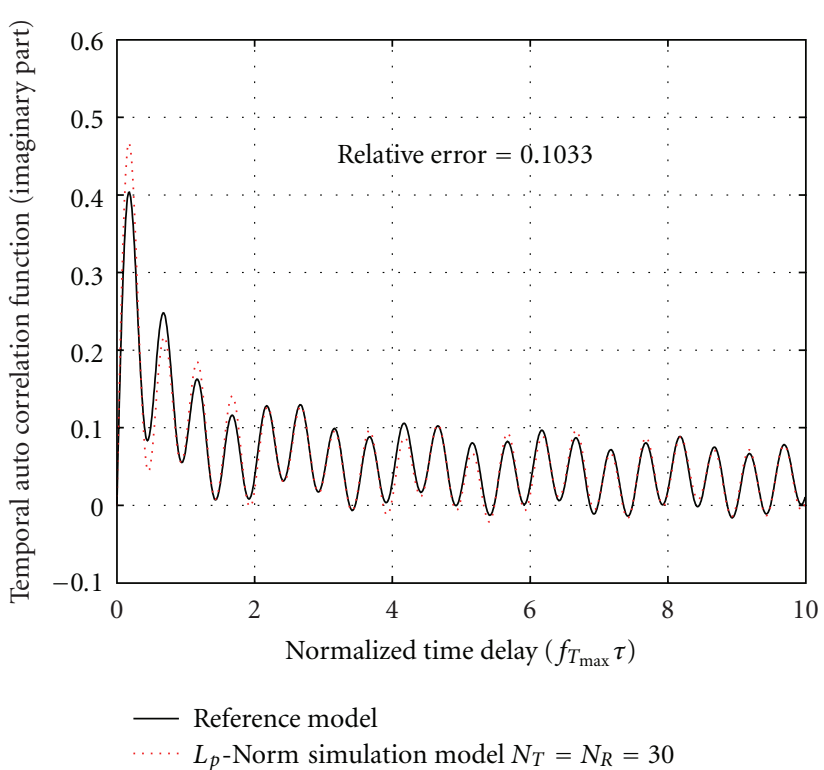

(b)

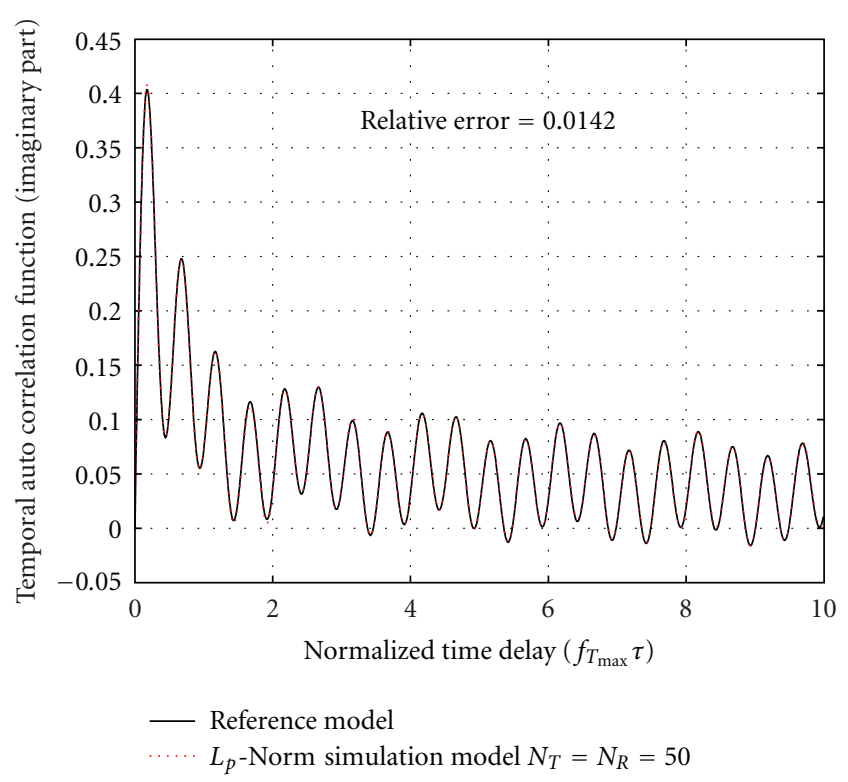

(d)

FIgURE 5: The normalized temporal ACF of the nonisotropic reference model and the $L_{p}$-Norm simulation model for $N_{T}=N_{R}=20,30,40$, 50 (imaginary part).

MEDS simulation model is negligible. The matched time is dependent on the number of scatterers and the relative error. It is denoted by $\left[f_{\max } \tau\right]_{\max }$, where $f_{\max }=f_{T_{\max }}=f_{R_{\max }}$. As evident from the simulations, the maximum time delay $\tau_{\max }$ is a key parameter for the proposed MEDS simulation model and requires to be set properly to use it for simulating the isotropic MGTR reference model. Also, by increasing the number of scatterers (the number of harmonic functions), $N_{T}$ and $N_{R}$, the matched time increases.

(ii) Second Scenario. In this scenario, we compare the STCCF of the simulation model $\left(\hat{\rho}_{l p, m q}(\tau)\right)$ with the
STCCF of the theoretical isotropic reference model $\left(\rho_{l p, m q}(\tau)\right)$ for $\delta_{T}^{p q}=\delta_{R}^{l m}=1 \lambda$. Figure 3 denotes this comparison for $\left\{N_{T}=N_{R}=20,30,40,50\right\}$. It is evident, like the first scenario, by increasing the number of scatterers (the number of harmonic functions), $N_{T}$ and $N_{R}$, the matched time increases.

6.2. The $L_{p}$-Norm Simulation Model. For performance evaluation of $L_{p}$-Norm simulation model, first we determine the optimum parameters $\varphi_{T}^{k}$ and $\phi_{R}^{i}$ by minimizing the error norm defined in (23). Note that in the following simulation scenarios, we assume $k_{T}=0.5$ and $k_{R}=0$, according to 


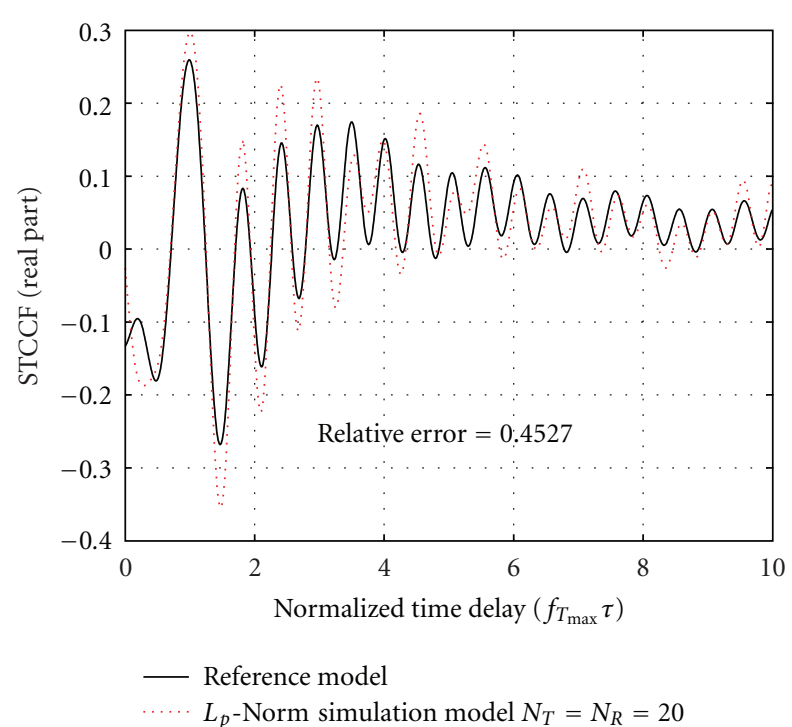

(a)

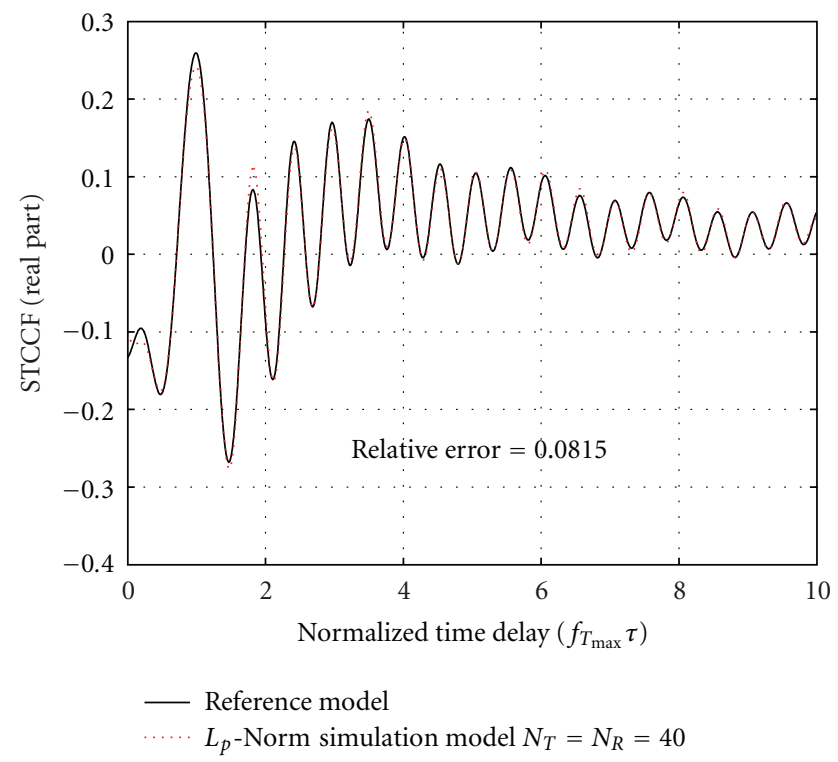

(c)

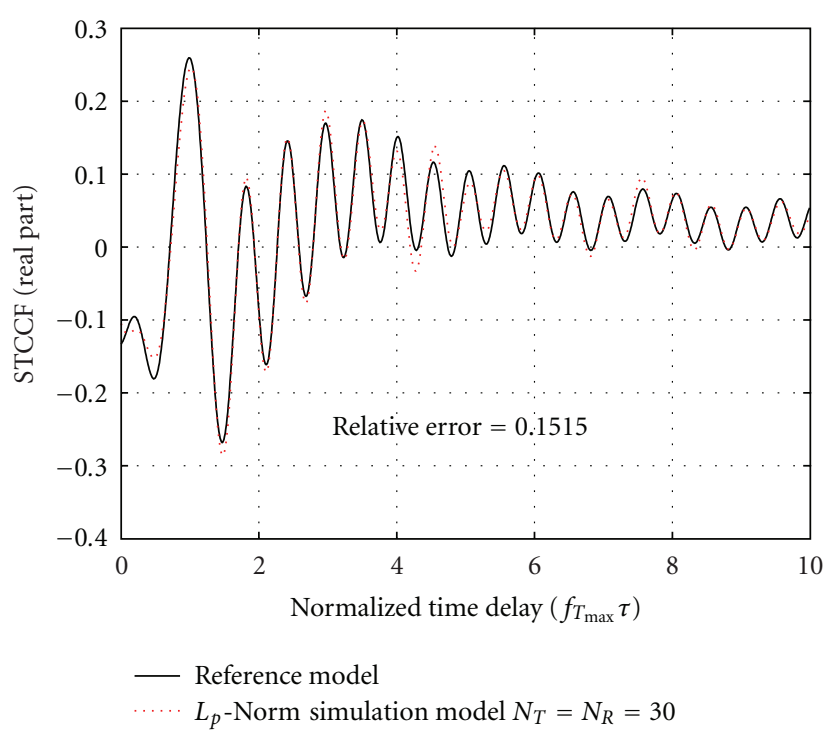

(b)

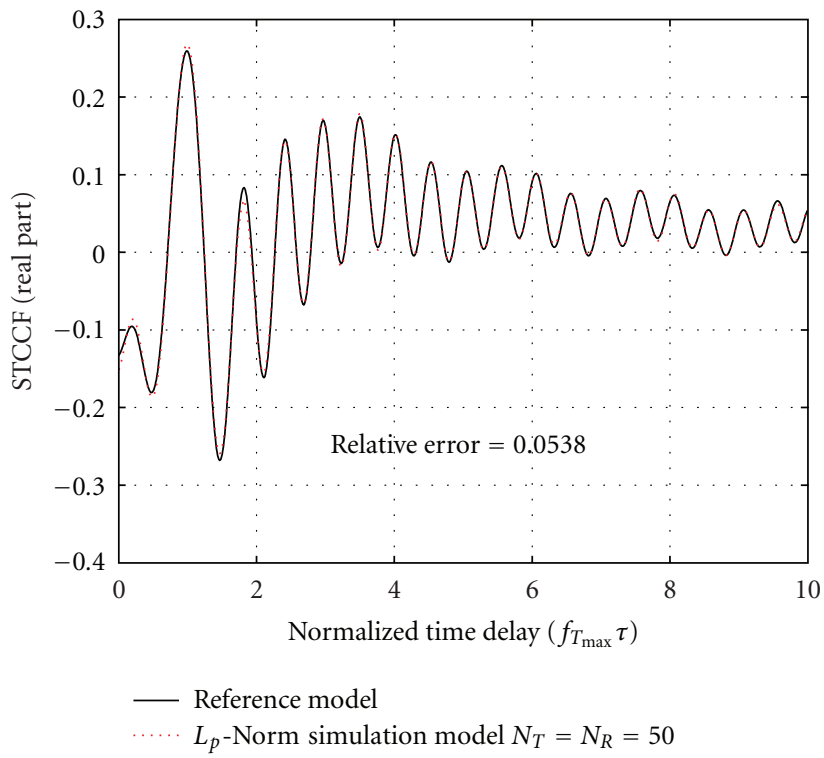

(d)

FIGURE 6: The STCCF of the nonisotropic reference model and the $L_{p}$-Norm simulation model for $\delta_{T}^{p q}=\delta_{R}^{l m}=1 \lambda$ and $N_{T}=N_{R}=20,30$, 40, 50 (real part).

Table I of [17]. Also, we assume $p=2$, actually, we minimize the $L_{2}$-Norm. Now, we consider two scenarios like the MEDS simulation model scenarios. First, we compare the temporal ACF of the $L_{p}$-Norm simulation model with the temporal ACF of theoretical nonisotropic reference model, derived in Section 3 (17) for $\left\{N_{T}=N_{R}=20,30,40,50\right\}$. Figures 4 and 5 show this comparison for real part and imaginary part of temporal ACF, respectively. Second, we compare the STCCF of the $L_{p}$-Norm simulation model with the STCCF of theoretical nonisotropic reference model, for $\delta_{T}^{p q}=\delta_{R}^{l m}=1 \lambda$ and $\left\{N_{T}=N_{R}=20,30,40,50\right\}$. This comparison result is shown in Figures 6 and 7 for real part and imaginary part of STCCF, respectively.
Note that the number displayed on the Figures $4-7$ is the minimized relative error of $L_{p}$-Norm method and defined as

$$
\text { relative error }=\frac{E_{\rho l p, m q}^{(2)}}{\left\{\left(1 / \tau_{\max }\right) \int_{0}^{\tau_{\max }}\left|\rho_{l p, m q}(\tau)\right|^{2} d \tau\right\}^{1 / 2}} .
$$

It must be mentioned, unlike the MEDS method, the $L_{p}$-Norm method has an advantage that can be applied to any given distribution of the local scatterers (it is useful for determining the nonuniform distributed discrete parameters AODs and AOAs). But, it has more complexity in comparison with the MEDS method. In MEDS method, 


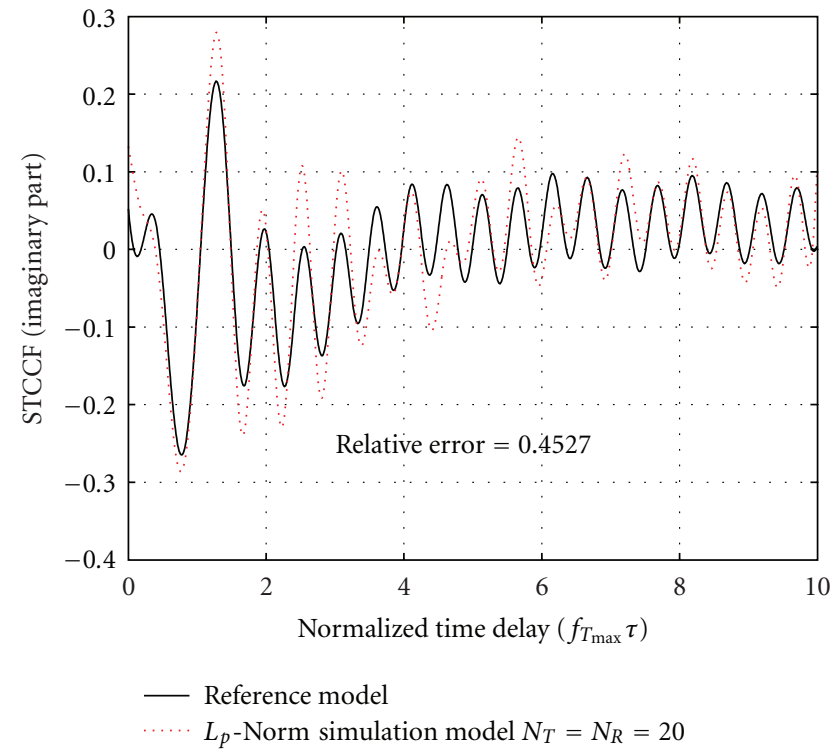

(a)

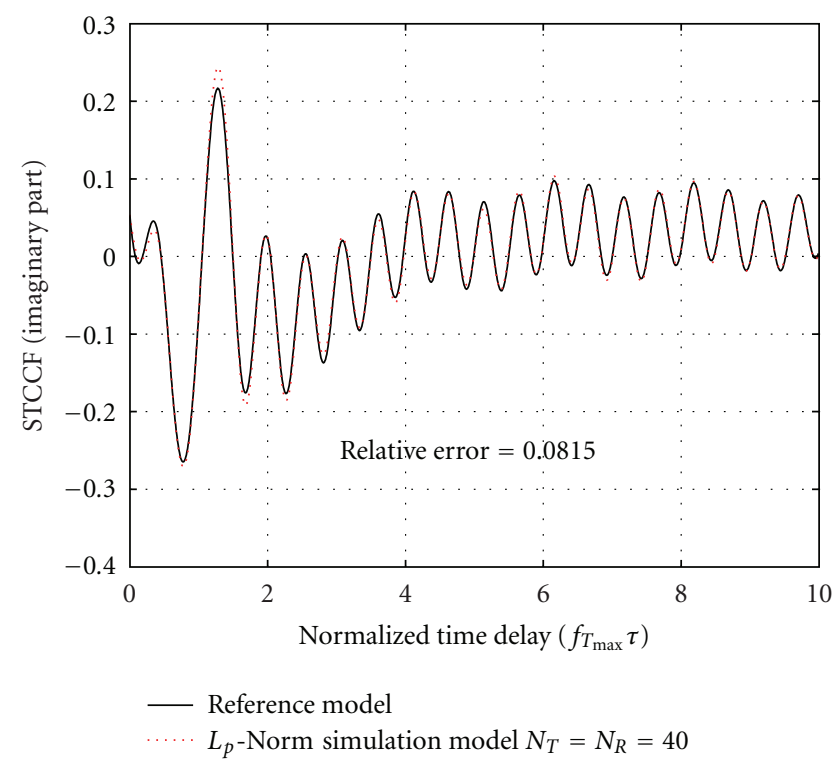

(c)

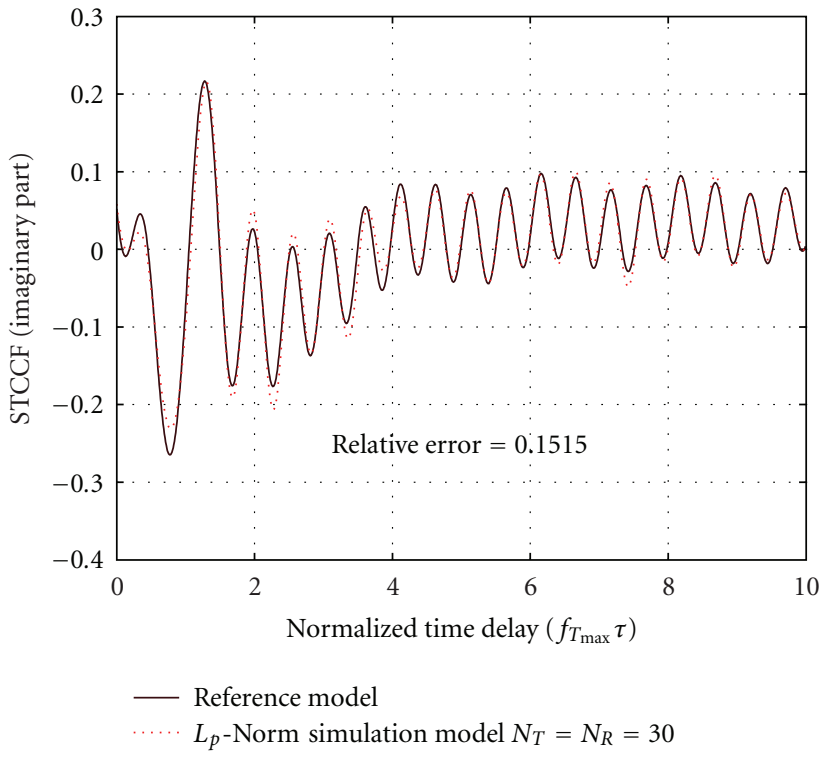

(b)

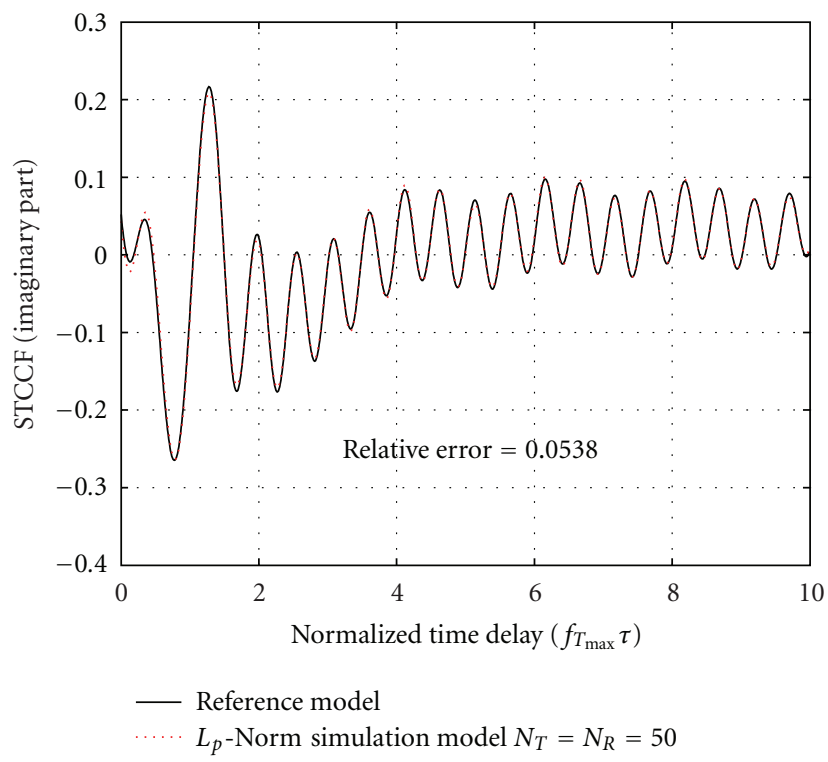

(d)

Figure 7: The STCCF of the nonisotropic reference model and the $L_{p}$-Norm simulation model for $\delta_{T}^{p q}=\delta_{R}^{l m}=1 \lambda$ and $N_{T}=N_{R}=20,30$, 40, 50 (imaginary part).

the Matched Time is depended on the relative error and the number of weighted harmonic functions (the number of scatterers around the transmitter and the receiver, $N_{T}$ and $N_{R}$ ). In the $L_{p}$-Norm method, the minimization is performed over interval $\left[0, \tau_{\max }\right]$ and the maximum Matched Time is equal to $\tau_{\max }$ for predefined constants $N_{T}$ and $N_{R}$ that by increasing them the minimization error is decreased.

\section{Conclusion}

This paper proposed a theoretical reference model for Rayleigh fading MIMO M-to-M channels. This reference model was based on the extension of single-bounce tworing model that avoids the technical difficulties of the double-bounce two-ring model. The closed-form crosscorrelation function for $2 \mathrm{D}$ nonisotropic scattering was derived for this proposed reference model. The presented model is an extension of M-to-M channel model proposed by Akki and Haber with respect to multiple antenna at the transmitter and the receiver. Moreover, it includes the single-bounce two-ring MIMO channel model introduced by Wang et al. as a special case when the transmitter is fixed and only the receiver is moving. Also, we propose two efficient and realizable statistical simulation models for 
simulating the theoretical reference model in both isotropic and nonisotropic conditions. The correctness of proposed simulation models was shown via different simulation trials.

\section{Acknowledgment}

The financial support from the Iran Telecommunication Research Center (ITRC) is gratefully acknowledged.

\section{References}

[1] D. S. Shiu, G. J. Foschini, M. J. Gans, and J. M. Kahn, "Fading correlation and its effect on the capacity of multielement antenna systems," IEEE Transactions on Communications, vol. 48, no. 3, pp. 502-513, 2000.

[2] A. Abdi and M. Kaveh, "A space-time correlation model for multielement antenna systems in mobile fading channels," IEEE Journal on Selected Areas in Communications, vol. 20, no. 3, pp. 550-560, 2002.

[3] M. Steinbauer, D. Hampicke, G. Sommerkorn et al., "Array measurement of the doubledirectional mobile radio channel," in Proceedings of the IEEE Vehicular Technology Conference, pp. 1656-1662, Tokyo, Japan, 2000.

[4] M. Steinbauer, A. F. Molisch, and E. Bonek, "The doubledirectional radio channel," IEEE Antennas and Propagation Magazine, vol. 43, no. 4, pp. 51-63, 2001.

[5] D. Gesbert, H. Bölcskei, D. A. Gore, and A. J. Paulraj, "Outdoor MIMO wireless channels: models and performance prediction," IEEE Transactions on Communications, vol. 50, no. 12, pp. 1926-1934, 2002.

[6] A. S. Akki and F. Haber, "A statistical model of radio mobileto-mobile land communication channel," IEEE Transactions on Vehicular Technology, vol. 35, no. 1, pp. 2-7, 1986.

[7] A. S. Akki, "Statistical properties of mobile-to-mobile land communication channels," IEEE Transactions on Vehicular Technology, vol. 43, no. 4, pp. 826-831, 1994.

[8] C. S. Patel, G. L. Stüber, and T. G. Pratt, "Simulation of Rayleigh-faded mobile-to-mobile communication channels," IEEE Transactions on Communications, vol. 53, no. 11, pp. 1876-1884, 2005.

[9] A. G. Zajić and G. L. Stüber, "A new simulation model for mobile-to-mobile rayleigh fading channels," in Proceedings of the IEEE Wireless Communications and Networking Conference (WCNC'06), pp. 1266-1270, Las Vegas, Nev, USA, April 2006.

[10] M. Pätzold, B. O. Hogstad, N. Youssef, and D. Kim, "A MIMO mobileto-mobile channel model: part I-the reference model," in Proceedings of the Personal, Indoor and Mobile Radio Communications (PIMRC '05), pp. 573-578, Berlin, Germany, September 2005.

[11] B. O. Hogstad, M. Pätzold, N. Youssef, and D. Kim, "A MIMO mobileto-mobile channel model: part II-the simulation model," in Proceedings of the Personal, Indoor and Mobile Radio Communications (PIMRC '05), pp. 562-567, Berlin, Germany, September 2005.

[12] M. Pätzold, B. O. Hogstad, and N. Youssef, "Modeling, analysis, and simulation of MIMO mobile-to-mobile fading channels," IEEE Transactions on Wireless Communications, vol. 7, no. 2, pp. 510-520, 2008.

[13] A. G. Zajić and G. L. Stüber, "Space-time correlated MIMO mobile-to-mobile channels," in Proceedings of the IEEE 17th International Symposium on Personal, Indoor and Mobile Radio
Communications (PIMRC '06), Helsinki, Finland, September 2006.

[14] A. G. Zajić and G. L. Stüber, "Simulation models for MIMO mobileto-mobile channels," in Proceedings of the IEEE Military Communications Conference (MILCOM '06), pp. 1-7, Washington, DC, USA, October 2006.

[15] K. Yu and B. Ottersten, "Models for MIMO propagation channels: a review," Wireless Communications and Mobile Computing, vol. 2, no. 7, pp. 653-666, 2002.

[16] K. Yu, Multiple-input multiple-output radio propagation channels: characteristics and models, Doctoral thesis, Signals, Sensors and Systems, Royal Institute of Technology (KTH), 2005.

[17] S. Wang, A. Abdi, J. Salo et al., "Time-varying MIMO channels: parametric statistical modeling and experimental results," IEEE Transactions on Vehicular Technology, vol. 56, no. 4, pp. 1949-1963, 2007.

[18] A. Abdi, J. A. Barger, and M. Kaveh, "A parametric model for the distribution of the angle of arrival and the associated correlation function and power spectrum at the mobile station," IEEE Transactions on Vehicular Technology, vol. 51, no. 3, pp. 425-434, 2002.

[19] I. S. Gradshteyn and I. M. Ryzhik, "Table of Integral, Series and Products," A. Jeffrey, Ed., Academic Press, San Diego, Calif, USA, 5th edition, 1994.

[20] G. Bakhshi, R. Saadat, and K. Shahtalebi, "A modified two-ring reference model for MIMO mobile-to-mobile communication channels," in Proceedings of the International Symposium on Telecommunications (IST '08), pp. 409-413, Tehran, Iran, August 2008.

[21] R. H. Clarke, "A statistical theory of mobile-radio reception," Bell System Technical Journal, vol. 47, pp. 957-1000, 1968.

[22] M. Pätzold, Mobile Fading Channels, John Wiley \& Sons, Chichester, UK, 2002.

[23] M. Pätzold, U. Killat, F. Laue, and Y. Li, "On the statistical properties of deterministic simulation models for mobile fading channels," IEEE Transactions on Vehicular Technology, vol. 47, no. 1, pp. 254-269, 1998. 

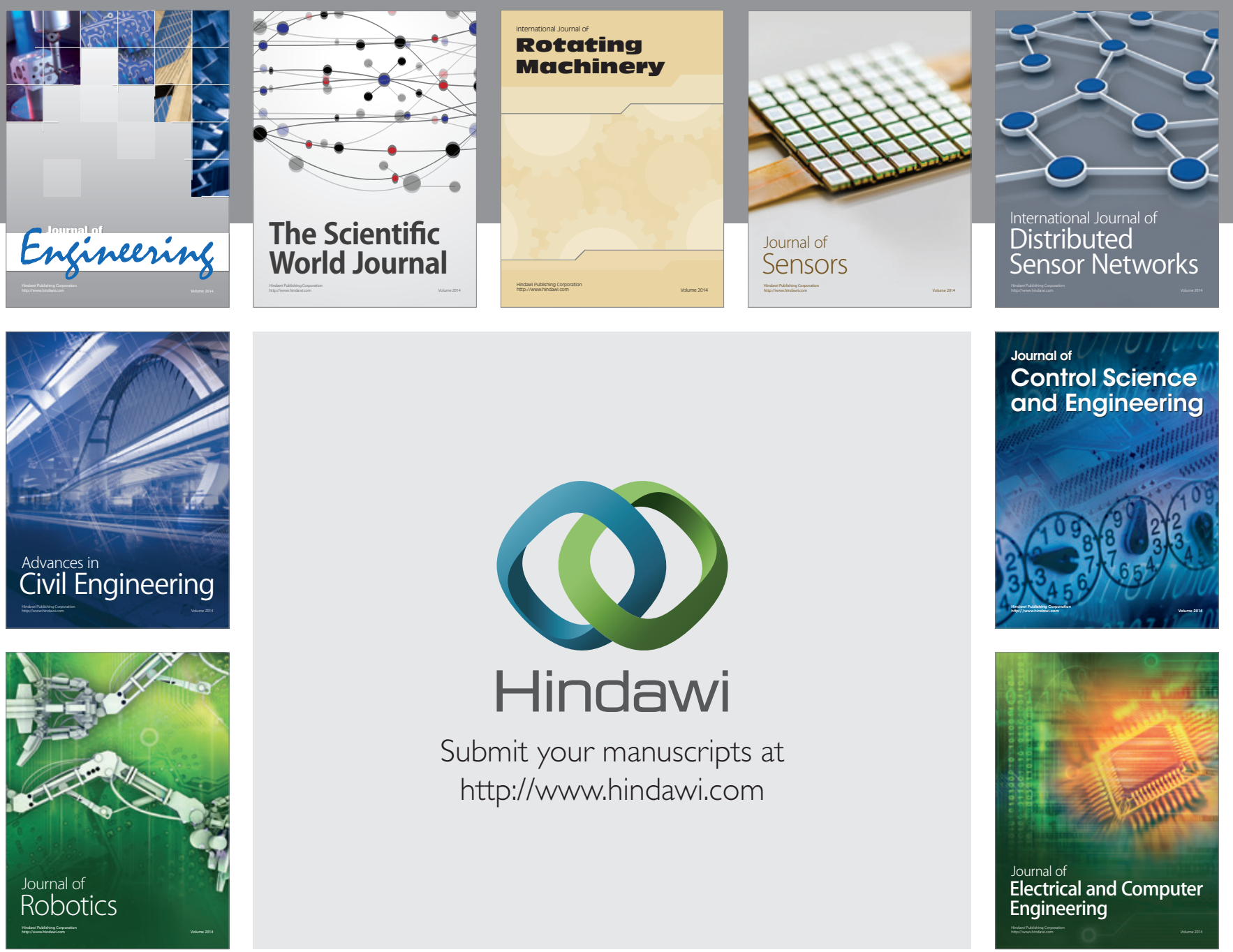

Submit your manuscripts at

http://www.hindawi.com
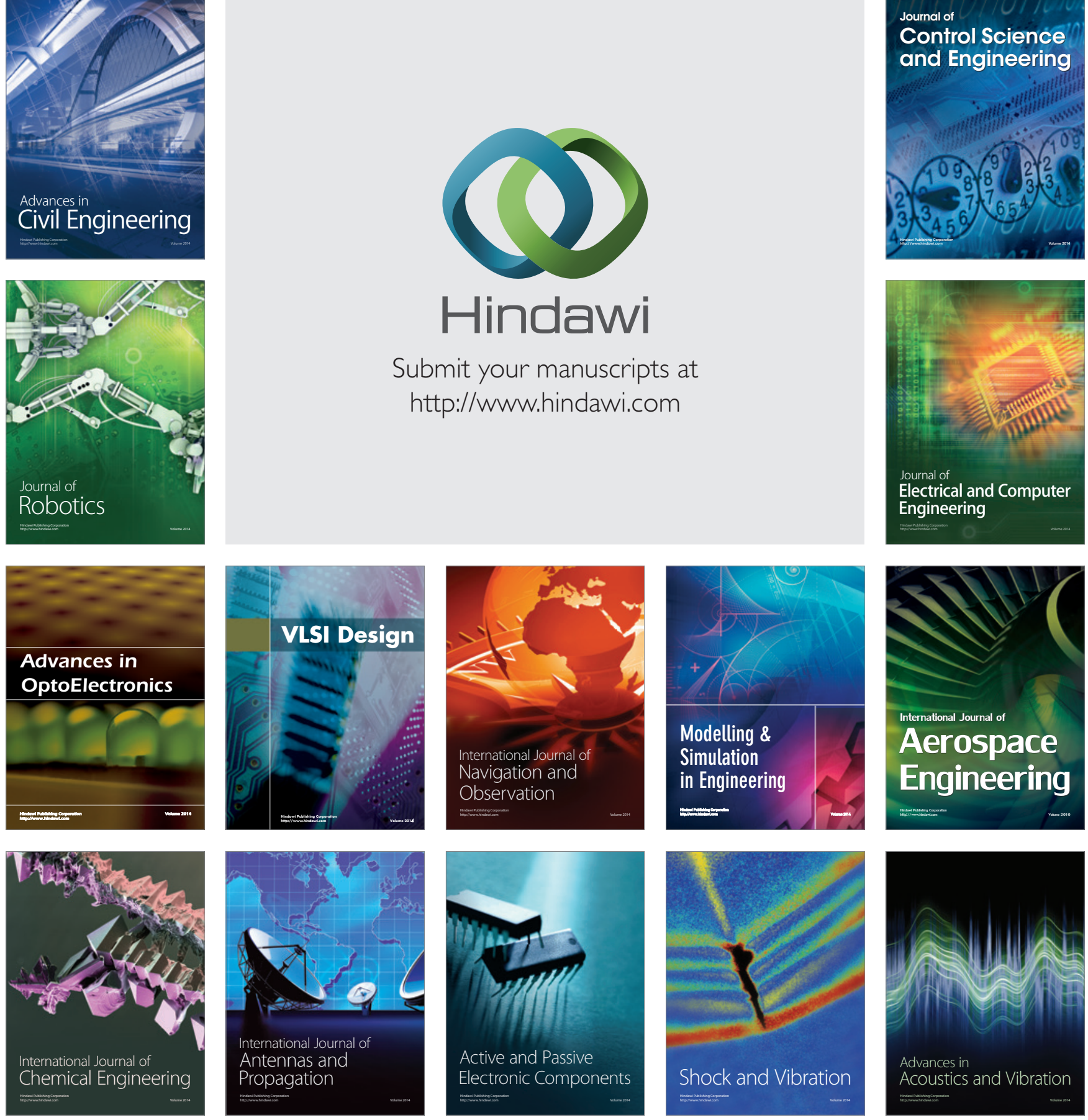